\title{
CRONOLOGÍA INFORMATIVA SOBRE LA PANDEMIA COVID EN ESPAÑA (ANTECEDENTES) ANEXO 3. WEBGRAFÍA
}

\section{Guillem Garcia Soler ${ }^{1}$}

Fuente: "La Vanguardia". Acceso abierto al público en general

\begin{tabular}{|c|c|c|}
\hline $\begin{array}{c}\text { Item ref. } \\
(*)\end{array}$ & Fecha & Enlace \\
\hline 1 & $15 / 2 / 2020$ & $\begin{array}{l}\text { http://hemeroteca.lavanguardia.com/preview/2020/04/25/pagina- } \\
\text { 18/289267447/pdf.html? search=coronavirus, } \% 20 \text { febrero } \% 202020\end{array}$ \\
\hline 2 & $15 / 2 / 2020$ & $\begin{array}{l}\text { http://hemeroteca.lavanguardia.com/preview/2020/02/15/pagina- } \\
\text { 19/289267146/pdf.html }\end{array}$ \\
\hline 3 & $15 / 2 / 2020$ & $\begin{array}{l}\text { http://hemeroteca.lavanguardia.com/preview/2020/02/15/pagina- } \\
\text { 26/289267459/pdf.html?search=coronavirus }\end{array}$ \\
\hline 4 & $15 / 2 / 2020$ & $\begin{array}{l}\text { http://hemeroteca.lavanguardia.com/preview/2020/02/11/pagina- } \\
\text { 50/289267558/pdf.html? } \text { search=coronavirus, } \% 20 \text { febrero } \% 202020\end{array}$ \\
\hline 5 & $16 / 2 / 2020$ & $\begin{array}{l}\text { http://hemeroteca.lavanguardia.com/preview/2020/02/16/pagina- } \\
\text { 8/289616559/pdf.html?search=coronavirus }\end{array}$ \\
\hline 6 & $16 / 2 / 2020$ & $\begin{array}{l}\text { http://hemeroteca.lavanguardia.com/preview/2020/02/16/pagina- } \\
\text { 29/289616144/pdf.html?search=coronavirus }\end{array}$ \\
\hline 7 & $16 / 2 / 2020$ & $\begin{array}{l}\text { http://hemeroteca.lavanguardia.com/preview/2020/02/16/pagina- } \\
\text { 34/289616428/pdf.html? } \text { search=coronavirus, } \% 20 \text { febrero } \% 202020\end{array}$ \\
\hline 8 & $16 / 2 / 2020$ & $\begin{array}{l}\text { http://hemeroteca.lavanguardia.com/preview/2020/02/13/pagina- } \\
\text { 74/289616083/pdf.html?search=coronavirus, } \% 20 \text { febrero } \% 202020\end{array}$ \\
\hline 9 & $17 / 2 / 2020$ & $\begin{array}{l}\text { http://hemeroteca.lavanguardia.com/preview/2020/02/25/pagina- } \\
\text { 1/289767300/pdf.html?search=coronavirus }\end{array}$ \\
\hline
\end{tabular}

${ }^{1}$ Investigador independiente guillem.garcia@telefonica.net 


\begin{tabular}{|c|c|c|}
\hline Item ref. & Fecha & Enlace \\
\hline 10 & $17 / 2 / 2020$ & $\begin{array}{l}\text { http://hemeroteca.lavanguardia.com/preview/2020/02/16/pagina- } \\
\text { 24/289766751/pdf.html?search=coronavirus, } \% 20 \text { febrero } \% 202020\end{array}$ \\
\hline 11 & $17 / 2 / 2020$ & $\begin{array}{l}\text { http://hemeroteca.lavanguardia.com/preview/2020/03/13/pagina- } \\
\text { 26/289767312/pdf.html?search=coronavirus, } \% 20 \text { febrero } \% 202020\end{array}$ \\
\hline 12 & $17 / 2 / 2020$ & $\begin{array}{l}\text { http://hemeroteca.lavanguardia.com/preview/2020/03/31/pagina- } \\
27 / 289767324 / \text { pdf.html? search=coronavirus, } \% 20 \text { febrero } \% 202020\end{array}$ \\
\hline 13 & $17 / 2 / 2020$ & $\begin{array}{l}\text { http://hemeroteca.lavanguardia.com/preview/2020/03/10/pagina- } \\
\text { 56/289766758/pdf.html?search=coronavirus, } \% 20 \text { febrero } \% 202020\end{array}$ \\
\hline 14 & $17 / 2 / 2020$ & $\begin{array}{l}\text { http://hemeroteca.lavanguardia.com/preview/2020/03/10/pagina- } \\
\text { 56/289766758/pdf.html?search=coronavirus, } \% 20 \text { febrero } \% 202020 \\
\end{array}$ \\
\hline 15 & $18 / 2 / 2020$ & $\begin{array}{l}\text { http://hemeroteca.lavanguardia.com/preview/2020/02/29/pagina- } \\
\text { 27/289967649/pdf.html?search=coronavirus, } \% 20 \text { febrero } \% 202020 \\
\end{array}$ \\
\hline 16 & $19 / 2 / 2020$ & $\begin{array}{l}\text { http://hemeroteca.lavanguardia.com/preview/2020/02/19/pagina- } \\
\text { 1/290117544/pdf.html }\end{array}$ \\
\hline 17 & $19 / 2 / 2020$ & $\begin{array}{l}\text { http://hemeroteca.lavanguardia.com/preview/2020/02/09/pagina- } \\
\text { 25/290117860/pdf.html?search=coronavirus, } \% 20 \text { febrero } \% 202020\end{array}$ \\
\hline 18 & $20 / 2 / 2020$ & $\begin{array}{l}\text { http://hemeroteca.lavanguardia.com/preview/2020/02/25/pagina- } \\
1 / 290517478 / \text { pdf.html? search=coronavirus }\end{array}$ \\
\hline 19 & $20 / 2 / 2020$ & $\begin{array}{l}\text { http://hemeroteca.lavanguardia.com/preview/2020/02/20/pagina- } \\
\text { 26/290517287/pdf.html?search=coronavirus, } \% 20 \text { febrero } \% 202020\end{array}$ \\
\hline 20 & $21 / 2 / 2020$ & $\begin{array}{l}\text { http://hemeroteca.lavanguardia.com/preview/2020/02/16/pagina- } \\
\text { 20/290816799/pdf.html?search=coronavirus, } \% 20 \text { febrero } \% 202020\end{array}$ \\
\hline 21 & $21 / 2 / 2020$ & $\begin{array}{l}\text { http://hemeroteca.lavanguardia.com/preview/2020/04/05/pagina- } \\
\text { 24/290816913/pdf.html?search=coronavirus, } \% 20 \text { febrero } \% 202020\end{array}$ \\
\hline 22 & $21 / 2 / 2020$ & $\begin{array}{l}\text { http://hemeroteca.lavanguardia.com/preview/2020/02/14/pagina- } \\
\text { 50/290817231/pdf.html? search=coronavirus, } \% 20 \text { febrero } \% 202020\end{array}$ \\
\hline 23 & $22 / 2 / 2020$ & $\begin{array}{l}\text { http://hemeroteca.lavanguardia.com/preview/2020/03/15/pagina- } \\
\text { 1/291068351/pdf.html?search=coronavirus }\end{array}$ \\
\hline 24 & $22 / 2 / 2020$ & $\begin{array}{l}\text { http://hemeroteca.lavanguardia.com/preview/2020/02/06/pagina- } \\
\text { 20/291067452/pdf.html?search=coronavirus, } \% 20 \text { febrero } \% 202020\end{array}$ \\
\hline 25 & $22 / 2 / 2020$ & $\begin{array}{l}\text { http://hemeroteca.lavanguardia.com/preview/2020/02/22/pagina- } \\
\text { 24/291067151/pdf.html? search=coronavirus, } \% 20 \text { febrero } \% 202020\end{array}$ \\
\hline 26 & $22 / 2 / 2020$ & $\begin{array}{l}\text { http://hemeroteca.lavanguardia.com/preview/2020/02/22/pagina- } \\
\text { 26/291068386/pdf.html }\end{array}$ \\
\hline 27 & $22 / 2 / 2020$ & $\begin{array}{l}\text { http://hemeroteca.lavanguardia.com/preview/2020/02/22/pagina- } \\
\text { 27/291068381/pdf.html }\end{array}$ \\
\hline 28 & $23 / 2 / 2020$ & $\begin{array}{l}\text { http://hemeroteca.lavanguardia.com/preview/2020/02/16/pagina- } \\
11 / 291066754 / \text { pdf.html? search=coronavirus, } \% 20 \text { febrero } \% 202020\end{array}$ \\
\hline
\end{tabular}




\begin{tabular}{|c|c|c|}
\hline Item ref. & Fecha & Enlace \\
\hline 29 & $23 / 2 / 2020$ & $\begin{array}{l}\text { http://hemeroteca.lavanguardia.com/preview/2020/02/23/pagina- } \\
\text { 32/291167920/pdf.html }\end{array}$ \\
\hline 30 & $23 / 2 / 2020$ & $\begin{array}{l}\text { http://hemeroteca.lavanguardia.com/preview/2020/02/23/pagina- } \\
\text { 33/291167915/pdf.html }\end{array}$ \\
\hline 31 & $23 / 2 / 2020$ & $\begin{array}{l}\text { http://hemeroteca.lavanguardia.com/preview/2020/02/13/pagina- } \\
\text { 70/291167331/pdf.html?search=coronavirus, } \% 20 \text { febrero } \% 202020\end{array}$ \\
\hline 32 & $24 / 2 / 2020$ & $\begin{array}{l}\text { http://hemeroteca.lavanguardia.com/preview/2020/02/25/pagina- } \\
\text { 1/291417561/pdf.html?search=coronavirus }\end{array}$ \\
\hline 33 & $24 / 2 / 2020$ & $\begin{array}{l}\text { http://hemeroteca.lavanguardia.com/preview/2020/02/18/pagina- } \\
\text { 27/291417639/pdf.html?search=coronavirus }\end{array}$ \\
\hline 34 & $24 / 2 / 2020$ & $\begin{array}{l}\text { http://hemeroteca.lavanguardia.com/preview/2020/02/18/pagina- } \\
\text { 27/291417639/pdf.html?search=coronavirus }\end{array}$ \\
\hline 35 & $24 / 2 / 2020$ & $\begin{array}{l}\text { http://hemeroteca.lavanguardia.com/preview/2020/02/21/pagina- } \\
\text { 28/291417615/pdf.html?search= coronavirus }\end{array}$ \\
\hline 36 & $24 / 2 / 2020$ & $\begin{array}{l}\text { http://hemeroteca.lavanguardia.com/preview/2020/02/11/pagina- } \\
\text { 54/291417245/pdf.html?search=coronavirus, } \% 20 \text { febrero } \% 202020\end{array}$ \\
\hline 37 & $25 / 2 / 2020$ & $\begin{array}{l}\text { http://hemeroteca.lavanguardia.com/preview/2020/02/19/pagina- } \\
\text { 1/291564950/pdf.html?search=coronavirus }\end{array}$ \\
\hline 38 & $25 / 2 / 2020$ & $\begin{array}{l}\text { http://hemeroteca.lavanguardia.com/preview/2020/02/06/pagina- } \\
\text { 20/291564477/pdf.html?search=coronavirus, } \% 20 \text { febrero } \% 202020\end{array}$ \\
\hline 39 & $25 / 2 / 2020$ & $\begin{array}{l}\text { http://hemeroteca.lavanguardia.com/preview/2020/02/25/pagina- } \\
\text { 24/291564909/pdf.html }\end{array}$ \\
\hline 40 & $25 / 2 / 2020$ & $\begin{array}{l}\text { http://hemeroteca.lavanguardia.com/preview/2020/02/25/pagina- } \\
\text { 25/291564913/pdf.html }\end{array}$ \\
\hline 41 & $25 / 2 / 2020$ & $\begin{array}{l}\text { http://hemeroteca.lavanguardia.com/preview } / 2020 / 02 / 07 / \text { pagina- } \\
\text { 26/291564921/pdf.html? search=coronavirus, } \% 20 \text { febrero } \% 202020\end{array}$ \\
\hline 42 & $25 / 2 / 2020$ & $\begin{array}{l}\text { http://hemeroteca.lavanguardia.com/preview/2020/02/07/pagina- } \\
\text { 26/291564921/pdf.html? search=coronavirus, } \% 20 \text { febrero } \% 202020\end{array}$ \\
\hline 43 & $25 / 2 / 2020$ & $\begin{array}{l}\text { http://hemeroteca.lavanguardia.com/preview/2020/02/18/pagina- } \\
\text { 27/291564582/pdf.html?search=coronavirus }\end{array}$ \\
\hline 44 & $25 / 2 / 2020$ & $\begin{array}{l}\text { http://hemeroteca.lavanguardia.com/preview/2020/02/25/pagina- } \\
\text { 46/291564963/pdf.html }\end{array}$ \\
\hline 45 & $26 / 2 / 2020$ & $\begin{array}{l}\text { http://hemeroteca.lavanguardia.com/preview/2020/02/26/pagina- } \\
\text { 1/291669045/pdf.html?search=coronavirus }\end{array}$ \\
\hline 46 & $26 / 2 / 2020$ & $\begin{array}{l}\text { http://hemeroteca.lavanguardia.com/preview/2020/02/13/pagina- } \\
\text { 2/291668983/pdf.html?search=coronavirus, } \% 20 \text { febrero } \% 202020\end{array}$ \\
\hline 47 & $26 / 2 / 2020$ & $\begin{array}{l}\text { http://hemeroteca.lavanguardia.com/preview/2020/02/26/pagina- } \\
\text { 22/291668998/pdf.html? search=coronavirus, } \% 20 \text { febrero } \% 202020\end{array}$ \\
\hline
\end{tabular}




\begin{tabular}{|c|c|c|}
\hline Item ref. & Fecha & Enlace \\
\hline 48 & $26 / 2 / 2020$ & $\begin{array}{l}\text { http://hemeroteca.lavanguardia.com/preview/2020/02/20/pagina- } \\
\text { 24/291668333/pdf.html?search=coronavirus, } \% 20 \text { febrero } \% 202020\end{array}$ \\
\hline 49 & $26 / 2 / 2020$ & $\begin{array}{l}\text { http://hemeroteca.lavanguardia.com/preview/2020/02/28/pagina- } \\
\text { 25/291669026/pdf.html?search=coronavirus, } \% 20 \text { febrero } \% 202020\end{array}$ \\
\hline 50 & $26 / 2 / 2020$ & $\begin{array}{l}\text { http://hemeroteca.lavanguardia.com/preview/2020/03/31/pagina- } \\
\text { 26/291668623/pdf.html? search=coronavirus, } \% 20 \text { febrero } \% 202020 \\
\end{array}$ \\
\hline 51 & $26 / 2 / 2020$ & $\begin{array}{l}\text { http://hemeroteca.lavanguardia.com/preview/2020/04/16/pagina- } \\
\text { 27/291668538/pdf.html?search=coronavirus, } \% 20 \text { febrero } \% 202020 \\
\end{array}$ \\
\hline 52 & $26 / 2 / 2020$ & $\begin{array}{l}\text { http://hemeroteca.lavanguardia.com/preview/2020/03/19/pagina- } \\
\text { 45/291668683/pdf.html?search=coronavirus, } \% 20 \text { febrero } \% 202020\end{array}$ \\
\hline 53 & $26 / 2 / 2020$ & $\begin{array}{l}\text { http://hemeroteca.lavanguardia.com/preview/2020/02/10/pagina- } \\
\text { 48/291668389/pdf.html? search=coronavirus, } \% 20 \text { febrero } \% 202020\end{array}$ \\
\hline 54 & $27 / 2 / 2020$ & $\begin{array}{l}\text { http://hemeroteca.lavanguardia.com/preview/2020/03/01/pagina- } \\
\text { 1/291817533/pdf.html?search=coronavirus }\end{array}$ \\
\hline 55 & $27 / 2 / 2020$ & $\begin{array}{l}\text { http://hemeroteca.lavanguardia.com/preview/2020/02/27/pagina- } \\
\text { 6/291817019/pdf.html? search=coronavirus, } \% 20 \text { febrero } \% 202020\end{array}$ \\
\hline 56 & $27 / 2 / 2020$ & $\begin{array}{l}\text { http://hemeroteca.lavanguardia.com/preview/2020/04/16/pagina- } \\
\text { 26/291817197/pdf.html?search=coronavirus, } \% 20 \text { febrero } \% 202020\end{array}$ \\
\hline 57 & $27 / 2 / 2020$ & $\begin{array}{l}\text { http://hemeroteca.lavanguardia.com/preview/2020/02/22/pagina- } \\
\text { 27/291817220/pdf.html?search=coronavirus, } \% 20 \text { febrero } \% 202020\end{array}$ \\
\hline 58 & $27 / 2 / 2020$ & $\begin{array}{l}\text { http://hemeroteca.lavanguardia.com/preview/2020/02/22/pagina- } \\
\text { 27/291817220/pdf.html?search=coronavirus, } \% 20 \text { febrero } \% 202020\end{array}$ \\
\hline 59 & $27 / 2 / 2020$ & $\begin{array}{l}\text { http://hemeroteca.lavanguardia.com/preview/2020/02/27/pagina- } \\
\text { 28/291817204/pdf.html }\end{array}$ \\
\hline 60 & $27 / 2 / 2020$ & $\begin{array}{l}\text { http://hemeroteca.lavanguardia.com/preview/2020/02/27/pagina- } \\
\text { 28/291817204/pdf.html }\end{array}$ \\
\hline 61 & $27 / 2 / 2020$ & $\begin{array}{l}\text { http://hemeroteca.lavanguardia.com/preview/2020/02/11/pagina- } \\
\text { 29/291817296/pdf.html? search=coronavirus, } \% 20 \text { febrero } \% 202020 \\
\end{array}$ \\
\hline 62 & $27 / 2 / 2020$ & $\begin{array}{l}\text { http://hemeroteca.lavanguardia.com/preview/2020/04/16/pagina- } \\
\text { 30/291817288/pdf.html?search=coronavirus, } \% 20 \text { febrero } \% 202020 \\
\end{array}$ \\
\hline 63 & $27 / 2 / 2020$ & $\begin{array}{l}\text { http://hemeroteca.lavanguardia.com/preview/2020/02/27/pagina- } \\
\text { 63/291817058/pdf.html }\end{array}$ \\
\hline 64 & $28 / 2 / 2020$ & $\begin{array}{l}\text { http://hemeroteca.lavanguardia.com/preview/2020/02/11/pagina- } \\
\text { 1/291967999/pdf.html? search=coronavirus, } \% 20 \text { febrero } \% 202020\end{array}$ \\
\hline 65 & $28 / 2 / 2020$ & $\begin{array}{l}\text { http://hemeroteca.lavanguardia.com/preview/2020/02/28/pagina- } \\
\text { 13/291967769/pdf.html }\end{array}$ \\
\hline 66 & $28 / 2 / 2020$ & $\begin{array}{l}\text { http://hemeroteca.lavanguardia.com/preview/2020/02/28/pagina- } \\
\text { 22/291967190/pdf.html }\end{array}$ \\
\hline
\end{tabular}




\begin{tabular}{|c|c|c|}
\hline $\begin{array}{c}\text { Item ref. } \\
(*)\end{array}$ & Fecha & Enlace \\
\hline 67 & $28 / 2 / 2020$ & $\begin{array}{l}\text { http://hemeroteca.lavanguardia.com/preview/2020/02/20/pagina- } \\
\text { 24/291967750/pdf.html?search=coronavirus, } \% 20 \text { febrero\%202020 }\end{array}$ \\
\hline 68 & $28 / 2 / 2020$ & $\begin{array}{l}\text { http://hemeroteca.lavanguardia.com/preview/2020/02/28/pagina- } \\
\text { 26/291967937/pdf.html }\end{array}$ \\
\hline 69 & $28 / 2 / 2020$ & $\begin{array}{l}\text { http://hemeroteca.lavanguardia.com/preview/2020/02/28/pagina- } \\
\text { 26/291967937/pdf.html }\end{array}$ \\
\hline 70 & $28 / 2 / 2020$ & $\begin{array}{l}\text { http://hemeroteca.lavanguardia.com/preview/2020/02/28/pagina- } \\
\text { 27/291967949/pdf.html }\end{array}$ \\
\hline 71 & $28 / 2 / 2020$ & $\begin{array}{l}\text { http://hemeroteca.lavanguardia.com/preview/2020/02/28/pagina- } \\
\text { 27/291967949/pdf.html }\end{array}$ \\
\hline 72 & $28 / 2 / 2020$ & $\begin{array}{l}\text { http://hemeroteca.lavanguardia.com/preview/2020/02/28/pagina- } \\
\text { 28/291967209/pdf.html }\end{array}$ \\
\hline 73 & $28 / 2 / 2020$ & $\begin{array}{l}\text { http://hemeroteca.lavanguardia.com/preview/2020/02/28/pagina- } \\
\text { 28/291967209/pdf.html }\end{array}$ \\
\hline 74 & $28 / 2 / 2020$ & $\begin{array}{l}\text { http://hemeroteca.lavanguardia.com/preview/2020/02/28/pagina- } \\
\text { 29/291967632/pdf.html }\end{array}$ \\
\hline 75 & $28 / 2 / 2020$ & $\begin{array}{l}\text { http://hemeroteca.lavanguardia.com/preview/2020/02/28/pagina- } \\
\text { 29/291967632/pdf.html }\end{array}$ \\
\hline 76 & $28 / 2 / 2020$ & $\begin{array}{l}\text { http://hemeroteca.lavanguardia.com/preview/2020/02/28/pagina- } \\
\text { 30/291967879/pdf.html }\end{array}$ \\
\hline 77 & $28 / 2 / 2020$ & $\begin{array}{l}\text { http://hemeroteca.lavanguardia.com/preview/2020/02/28/pagina- } \\
\text { 30/291967879/pdf.html }\end{array}$ \\
\hline 78 & $28 / 2 / 2020$ & $\begin{array}{l}\text { http://hemeroteca.lavanguardia.com/preview/2020/02/28/pagina- } \\
\text { 52/291967985/pdf.html }\end{array}$ \\
\hline 79 & $28 / 2 / 2020$ & $\begin{array}{l}\text { http://hemeroteca.lavanguardia.com/preview/2020/02/28/pagina- } \\
\text { 53/291967994/pdf.html }\end{array}$ \\
\hline 80 & $28 / 2 / 2020$ & $\begin{array}{l}\text { http://hemeroteca.lavanguardia.com/preview/2020/02/28/pagina- } \\
\text { 54/291967867/pdf.html }\end{array}$ \\
\hline 81 & $29 / 2 / 2020$ & $\begin{array}{l}\text { http://hemeroteca.lavanguardia.com/preview/2020/02/29/pagina- } \\
\text { 1/292117930/pdf.html }\end{array}$ \\
\hline 82 & $29 / 2 / 2020$ & $\begin{array}{l}\text { http://hemeroteca.lavanguardia.com/preview/2020/02/29/pagina- } \\
\text { 22/292117523/pdf.html }\end{array}$ \\
\hline 83 & $29 / 2 / 2020$ & $\begin{array}{l}\text { http://hemeroteca.lavanguardia.com/preview/2020/02/29/pagina- } \\
\text { 26/292118188/pdf.html }\end{array}$ \\
\hline 84 & $29 / 2 / 2020$ & $\begin{array}{l}\text { http://hemeroteca.lavanguardia.com/preview/2020/02/29/pagina- } \\
\underline{27 / 292118180 / \text { pdf.html }}\end{array}$ \\
\hline 85 & $29 / 2 / 2020$ & $\begin{array}{l}\text { http://hemeroteca.lavanguardia.com/preview/2020/02/29/pagina- } \\
\text { 27/292118180/pdf.html }\end{array}$ \\
\hline
\end{tabular}




\begin{tabular}{|c|c|c|}
\hline $\begin{array}{c}\text { Item ref. } \\
(*)\end{array}$ & Fecha & Enlace \\
\hline 86 & $29 / 2 / 2020$ & $\begin{array}{l}\text { http://hemeroteca.lavanguardia.com/preview/2020/02/29/pagina- } \\
\text { 28/292117949/pdf.html }\end{array}$ \\
\hline 87 & $29 / 2 / 2020$ & $\begin{array}{l}\text { http://hemeroteca.lavanguardia.com/preview/2020/02/29/pagina- } \\
\text { 28/292117949/pdf.html }\end{array}$ \\
\hline 88 & $29 / 2 / 2020$ & $\begin{array}{l}\text { http://hemeroteca.lavanguardia.com/preview/2020/02/29/pagina- } \\
\text { 50/292118035/pdf.html }\end{array}$ \\
\hline 89 & $29 / 2 / 2020$ & $\begin{array}{l}\text { http://hemeroteca.lavanguardia.com/preview/2020/02/29/pagina- } \\
\text { 51/292118051/pdf.html }\end{array}$ \\
\hline 90 & $29 / 2 / 2020$ & $\begin{array}{l}\text { http://hemeroteca.lavanguardia.com/preview/2020/02/29/pagina- } \\
\text { 52/292118059/pdf.html }\end{array}$ \\
\hline 91 & $1 / 3 / 2020$ & $\begin{array}{l}\text { http://hemeroteca.lavanguardia.com/preview/2020/03/01/pagina- } \\
\text { 1/292216729/pdf.html }\end{array}$ \\
\hline 92 & $1 / 3 / 2020$ & $\begin{array}{l}\text { http://hemeroteca.lavanguardia.com/preview/2020/03/01/pagina- } \\
\text { 2/292216925/pdf.html }\end{array}$ \\
\hline 93 & $1 / 3 / 2020$ & $\begin{array}{l}\text { http://hemeroteca.lavanguardia.com/preview/2020/03/01/pagina- } \\
\text { 28/292216639/pdf.html }\end{array}$ \\
\hline 94 & $1 / 3 / 2020$ & $\begin{array}{l}\text { http://hemeroteca.lavanguardia.com/preview/2020/03/01/pagina- } \\
\text { 30/292216745/pdf.html }\end{array}$ \\
\hline 95 & $1 / 3 / 2020$ & $\begin{array}{l}\text { http://hemeroteca.lavanguardia.com/preview/2020/03/01/pagina- } \\
\text { 30/292216745/pdf.html }\end{array}$ \\
\hline 96 & $1 / 3 / 2020$ & $\begin{array}{l}\text { http://hemeroteca.lavanguardia.com/preview/2020/03/01/pagina- } \\
\text { 33/292215983/pdf.html }\end{array}$ \\
\hline 97 & $1 / 3 / 2020$ & $\begin{array}{l}\text { http://hemeroteca.lavanguardia.com/preview/2020/03/01/pagina- } \\
\text { 34/292217165/pdf.html }\end{array}$ \\
\hline 98 & $1 / 3 / 2020$ & $\begin{array}{l}\text { http://hemeroteca.lavanguardia.com/preview/2020/03/01/pagina- } \\
\text { 35/292217177/pdf.html }\end{array}$ \\
\hline 99 & $1 / 3 / 2020$ & $\begin{array}{l}\text { http://hemeroteca.lavanguardia.com/preview/2020/03/01/pagina- } \\
\text { 35/292217177/pdf.html }\end{array}$ \\
\hline 100 & $1 / 3 / 2020$ & $\begin{array}{l}\text { http://hemeroteca.lavanguardia.com/preview/2020/03/01/pagina- } \\
\text { 36/292216986/pdf.html }\end{array}$ \\
\hline 101 & $1 / 3 / 2020$ & $\begin{array}{l}\text { http://hemeroteca.lavanguardia.com/preview/2020/03/01/pagina- } \\
\text { 36/292216986/pdf.html }\end{array}$ \\
\hline 102 & $1 / 3 / 2020$ & $\begin{array}{l}\text { http://hemeroteca.lavanguardia.com/preview/2020/03/01/pagina- } \\
\text { 37/292216990/pdf.html }\end{array}$ \\
\hline 103 & $1 / 3 / 2020$ & $\begin{array}{l}\text { http://hemeroteca.lavanguardia.com/preview/2020/03/01/pagina- } \\
\text { 37/292216990/pdf.html }\end{array}$ \\
\hline 104 & $1 / 3 / 2020$ & $\begin{array}{l}\text { http://hemeroteca.lavanguardia.com/preview/2020/03/01/pagina- } \\
\text { 48/292217005/pdf.html }\end{array}$ \\
\hline
\end{tabular}




\begin{tabular}{|c|c|c|}
\hline $\begin{array}{c}\text { Item ref. } \\
(*)\end{array}$ & Fecha & Enlace \\
\hline 105 & $1 / 3 / 2020$ & $\begin{array}{l}\text { http://hemeroteca.lavanguardia.com/preview/2020/03/01/pagina- } \\
\text { 68/292217110/pdf.html }\end{array}$ \\
\hline 106 & $1 / 3 / 2020$ & $\begin{array}{l}\text { http://hemeroteca.lavanguardia.com/preview/2020/03/01/pagina- } \\
\text { 72/292217102/pdf.html }\end{array}$ \\
\hline 107 & $1 / 3 / 2020$ & $\begin{array}{l}\text { http://hemeroteca.lavanguardia.com/preview/2020/03/01/pagina- } \\
\text { 72/292217102/pdf.html }\end{array}$ \\
\hline 108 & $1 / 3 / 2020$ & $\begin{array}{l}\text { http://hemeroteca.lavanguardia.com/preview/2020/03/01/pagina- } \\
\text { 73/292217149/pdf.html }\end{array}$ \\
\hline 109 & $2 / 3 / 2020$ & $\begin{array}{l}\text { http://hemeroteca.lavanguardia.com/preview/2020/03/02/pagina- } \\
\text { 1/292417594/pdf.html }\end{array}$ \\
\hline 110 & $2 / 3 / 2020$ & $\begin{array}{l}\text { http://hemeroteca.lavanguardia.com/preview/2020/03/02/pagina- } \\
\text { 21/292416868/pdf.html }\end{array}$ \\
\hline 111 & $2 / 3 / 2020$ & $\begin{array}{l}\text { http://hemeroteca.lavanguardia.com/preview/2020/03/02/pagina- } \\
\text { 26/292417155/pdf.html }\end{array}$ \\
\hline 112 & $2 / 3 / 2020$ & $\begin{array}{l}\text { http://hemeroteca.lavanguardia.com/preview/2020/03/02/pagina- } \\
\text { 27/292417147/pdf.html }\end{array}$ \\
\hline 113 & $2 / 3 / 2020$ & $\begin{array}{l}\text { http://hemeroteca.lavanguardia.com/preview/2020/03/02/pagina- } \\
\text { 27/292417147/pdf.html }\end{array}$ \\
\hline 114 & $2 / 3 / 2020$ & $\begin{array}{l}\text { http://hemeroteca.lavanguardia.com/preview/2020/03/02/pagina- } \\
27 / 292417147 / \text { pdf.html }\end{array}$ \\
\hline 115 & $2 / 3 / 2020$ & $\begin{array}{l}\text { http://hemeroteca.lavanguardia.com/preview/2020/03/02/pagina- } \\
\text { 27/292417147/pdf.html }\end{array}$ \\
\hline 116 & $2 / 3 / 2020$ & $\begin{array}{l}\text { http://hemeroteca.lavanguardia.com/preview/2020/03/02/pagina- } \\
\text { 28/292416888/pdf.html }\end{array}$ \\
\hline 117 & $2 / 3 / 2020$ & $\begin{array}{l}\text { http://hemeroteca.lavanguardia.com/preview/2020/03/02/pagina- } \\
\text { 29/292416900/pdf.html }\end{array}$ \\
\hline 118 & $2 / 3 / 2020$ & $\begin{array}{l}\text { http://hemeroteca.lavanguardia.com/preview/2020/03/02/pagina- } \\
\text { 53/292417329/pdf.html }\end{array}$ \\
\hline 119 & $2 / 3 / 2020$ & $\begin{array}{l}\text { http://hemeroteca.lavanguardia.com/preview/2020/03/02/pagina- } \\
\text { 57/292417499/pdf.html }\end{array}$ \\
\hline 120 & $2 / 3 / 2020$ & $\begin{array}{l}\text { http://hemeroteca.lavanguardia.com/preview/2020/03/02/pagina- } \\
\text { 57/292417499/pdf.html }\end{array}$ \\
\hline 121 & $3 / 3 / 2020$ & $\begin{array}{l}\text { http://hemeroteca.lavanguardia.com/preview/2020/03/03/pagina- } \\
\text { 1/292567339/pdf.html }\end{array}$ \\
\hline 122 & $3 / 3 / 2020$ & $\begin{array}{l}\text { http://hemeroteca.lavanguardia.com/preview/2020/03/03/pagina- } \\
\text { 1/292567339/pdf.html }\end{array}$ \\
\hline 123 & $3 / 3 / 2020$ & $\begin{array}{l}\text { http://hemeroteca.lavanguardia.com/preview/2020/03/02/pagina- } \\
\text { 2/292567321/pdf.html?search=coronavirus }\end{array}$ \\
\hline
\end{tabular}




\begin{tabular}{|c|c|c|}
\hline $\begin{array}{c}\text { Item ref. } \\
(*)\end{array}$ & Fecha & Enlace \\
\hline 124 & $3 / 3 / 2020$ & $\begin{array}{l}\text { http://hemeroteca.lavanguardia.com/preview/2020/03/03/pagina- } \\
\text { 18/292566966/pdf.html }\end{array}$ \\
\hline 125 & $3 / 3 / 2020$ & $\begin{array}{l}\text { http://hemeroteca.lavanguardia.com/preview/2020/03/03/pagina- } \\
\text { 19/292566735/pdf.html }\end{array}$ \\
\hline 126 & $3 / 3 / 2020$ & $\begin{array}{l}\text { http://hemeroteca.lavanguardia.com/preview/2020/03/03/pagina- } \\
\text { 22/292567425/pdf.html }\end{array}$ \\
\hline 127 & $3 / 3 / 2020$ & $\begin{array}{l}\text { http://hemeroteca.lavanguardia.com/preview/2020/03/03/pagina- } \\
\text { 23/292567433/pdf.html }\end{array}$ \\
\hline 128 & $3 / 3 / 2020$ & $\begin{array}{l}\text { http://hemeroteca.lavanguardia.com/preview/2020/03/03/pagina- } \\
\text { 23/292567433/pdf.html }\end{array}$ \\
\hline 129 & $3 / 3 / 2020$ & $\begin{array}{l}\text { http://hemeroteca.lavanguardia.com/preview/2020/03/03/pagina- } \\
\text { 24/292567609/pdf.html }\end{array}$ \\
\hline 130 & $3 / 3 / 2020$ & $\begin{array}{l}\text { http://hemeroteca.lavanguardia.com/preview/2020/03/03/pagina- } \\
\text { 24/292567609/pdf.html }\end{array}$ \\
\hline 131 & $3 / 3 / 2020$ & $\begin{array}{l}\text { http://hemeroteca.lavanguardia.com/preview/2020/03/03/pagina- } \\
\text { 25/292567605/pdf.html }\end{array}$ \\
\hline 132 & $3 / 3 / 2020$ & $\begin{array}{l}\text { http://hemeroteca.lavanguardia.com/preview/2020/03/03/pagina- } \\
\text { 33/292567469/pdf.html }\end{array}$ \\
\hline 133 & $3 / 3 / 2020$ & $\begin{array}{l}\text { http://hemeroteca.lavanguardia.com/preview/2020/03/03/pagina- } \\
\text { 44/292567245/pdf.html }\end{array}$ \\
\hline 134 & $3 / 3 / 2020$ & $\begin{array}{l}\text { http://hemeroteca.lavanguardia.com/preview/2020/03/03/pagina- } \\
\text { 45/292567241/pdf.html }\end{array}$ \\
\hline 135 & $3 / 3 / 2020$ & $\begin{array}{l}\text { http://hemeroteca.lavanguardia.com/preview/2020/03/03/pagina- } \\
\text { 46/292567228/pdf.html }\end{array}$ \\
\hline 136 & $3 / 3 / 2020$ & $\begin{array}{l}\text { http://hemeroteca.lavanguardia.com/preview/2020/03/03/pagina- } \\
\text { 46/292567228/pdf.html }\end{array}$ \\
\hline 137 & $4 / 3 / 2020$ & $\begin{array}{l}\text { http://hemeroteca.lavanguardia.com/preview/2020/03/04/pagina- } \\
\text { 1/292718017/pdf.html }\end{array}$ \\
\hline 138 & $4 / 3 / 2020$ & $\begin{array}{l}\text { http://hemeroteca.lavanguardia.com/preview/2020/03/04/pagina- } \\
\text { 1/292718017/pdf.html }\end{array}$ \\
\hline 139 & $4 / 3 / 2020$ & $\begin{array}{l}\text { http://hemeroteca.lavanguardia.com/preview/2020/03/04/pagina- } \\
\text { 2/292718032/pdf.html }\end{array}$ \\
\hline 140 & $4 / 3 / 2020$ & $\begin{array}{l}\text { http://hemeroteca.lavanguardia.com/preview/2020/03/04/pagina- } \\
\text { 22/292717783/pdf.html }\end{array}$ \\
\hline 141 & $4 / 3 / 2020$ & $\begin{array}{l}\text { http://hemeroteca.lavanguardia.com/preview/2020/03/04/pagina- } \\
\text { 23/292717795/pdf.html }\end{array}$ \\
\hline 142 & $4 / 3 / 2020$ & $\begin{array}{l}\text { http://hemeroteca.lavanguardia.com/preview/2020/03/04/pagina- } \\
\text { 23/292717795/pdf.html }\end{array}$ \\
\hline
\end{tabular}




\begin{tabular}{|c|c|c|}
\hline $\begin{array}{c}\text { Item ref. } \\
(*)\end{array}$ & Fecha & Enlace \\
\hline 143 & $4 / 3 / 2020$ & $\begin{array}{l}\text { http://hemeroteca.lavanguardia.com/preview/2020/03/04/pagina- } \\
\text { 24/292717504/pdf.html }\end{array}$ \\
\hline 144 & $4 / 3 / 2020$ & $\begin{array}{l}\text { http://hemeroteca.lavanguardia.com/preview/2020/03/04/pagina- } \\
\text { 24/292717504/pdf.html }\end{array}$ \\
\hline 145 & $4 / 3 / 2020$ & $\begin{array}{l}\text { http://hemeroteca.lavanguardia.com/preview/2020/03/04/pagina- } \\
\text { 39/292717336/pdf.html }\end{array}$ \\
\hline 146 & $4 / 3 / 2020$ & $\begin{array}{l}\text { http://hemeroteca.lavanguardia.com/preview/2020/03/04/pagina- } \\
\text { 40/292717340/pdf.html }\end{array}$ \\
\hline 147 & $4 / 3 / 2020$ & $\begin{array}{l}\text { http://hemeroteca.lavanguardia.com/preview/2020/03/04/pagina- } \\
\text { 44/292717645/pdf.html }\end{array}$ \\
\hline 148 & $4 / 3 / 2020$ & $\begin{array}{l}\text { http://hemeroteca.lavanguardia.com/preview/2020/03/04/pagina- } \\
\text { 45/292717649/pdf.html }\end{array}$ \\
\hline 149 & $5 / 3 / 2020$ & $\begin{array}{l}\text { http://hemeroteca.lavanguardia.com/preview/2020/03/05/pagina- } \\
\text { 1/292917915/pdf.html }\end{array}$ \\
\hline 150 & $5 / 3 / 2020$ & $\begin{array}{l}\text { http://hemeroteca.lavanguardia.com/preview/2020/03/05/pagina- } \\
\text { 1/292917915/pdf.html }\end{array}$ \\
\hline 151 & $5 / 3 / 2020$ & $\begin{array}{l}\text { http://hemeroteca.lavanguardia.com/preview/2020/03/05/pagina- } \\
\text { 1/292917915/pdf.html }\end{array}$ \\
\hline 152 & $5 / 3 / 2020$ & $\begin{array}{l}\text { http://hemeroteca.lavanguardia.com/preview/2020/03/05/pagina- } \\
\text { 22/292917290/pdf.html }\end{array}$ \\
\hline 153 & $5 / 3 / 2020$ & $\begin{array}{l}\text { http://hemeroteca.lavanguardia.com/preview/2020/03/05/pagina- } \\
\text { 24/292917860/pdf.html }\end{array}$ \\
\hline 154 & $5 / 3 / 2020$ & $\begin{array}{l}\text { http://hemeroteca.lavanguardia.com/preview/2020/03/05/pagina- } \\
\text { 25/292917864/pdf.html }\end{array}$ \\
\hline 155 & $5 / 3 / 2020$ & $\begin{array}{l}\text { http://hemeroteca.lavanguardia.com/preview/2020/03/05/pagina- } \\
\text { 25/292917864/pdf.html }\end{array}$ \\
\hline 156 & $5 / 3 / 2020$ & $\begin{array}{l}\text { http://hemeroteca.lavanguardia.com/preview/2020/03/05/pagina- } \\
\text { 26/292917868/pdf.html }\end{array}$ \\
\hline 157 & $5 / 3 / 2020$ & $\begin{array}{l}\text { http://hemeroteca.lavanguardia.com/preview/2020/03/05/pagina- } \\
\text { 27/292917888/pdf.html }\end{array}$ \\
\hline 158 & $5 / 3 / 2020$ & $\begin{array}{l}\text { http://hemeroteca.lavanguardia.com/preview/2020/03/05/pagina- } \\
\text { 45/292917651/pdf.html }\end{array}$ \\
\hline 159 & $5 / 3 / 2020$ & $\begin{array}{l}\text { http://hemeroteca.lavanguardia.com/preview/2020/03/05/pagina- } \\
\text { 52/292917830/pdf.html }\end{array}$ \\
\hline 160 & $5 / 3 / 2020$ & $\begin{array}{l}\text { http://hemeroteca.lavanguardia.com/preview/2020/03/05/pagina- } \\
\text { 52/292917830/pdf.html }\end{array}$ \\
\hline 161 & $5 / 3 / 2020$ & $\begin{array}{l}\text { http://hemeroteca.lavanguardia.com/preview/2020/03/05/pagina- } \\
\text { 53/292917826/pdf.html }\end{array}$ \\
\hline
\end{tabular}




\begin{tabular}{|c|c|c|}
\hline $\begin{array}{c}\text { Item ref. } \\
(*)\end{array}$ & Fecha & Enlace \\
\hline 162 & $5 / 3 / 2020$ & $\begin{array}{l}\text { http://hemeroteca.lavanguardia.com/preview/2020/03/05/pagina- } \\
\text { 53/292917826/pdf.html }\end{array}$ \\
\hline 163 & $6 / 3 / 2020$ & $\begin{array}{l}\text { http://hemeroteca.lavanguardia.com/preview/2020/03/06/pagina- } \\
\text { 1/293217122/pdf.html }\end{array}$ \\
\hline 164 & $6 / 3 / 2020$ & $\begin{array}{l}\text { http://hemeroteca.lavanguardia.com/preview/2020/03/06/pagina- } \\
\text { 2/293216860/pdf.html }\end{array}$ \\
\hline 165 & $6 / 3 / 2020$ & $\begin{array}{l}\text { http://hemeroteca.lavanguardia.com/preview/2020/03/06/pagina- } \\
\text { 20/293216762/pdf.html }\end{array}$ \\
\hline 166 & $6 / 3 / 2020$ & $\begin{array}{l}\text { http://hemeroteca.lavanguardia.com/preview/2020/03/06/pagina- } \\
\text { 21/293216776/pdf.html }\end{array}$ \\
\hline 167 & $6 / 3 / 2020$ & $\begin{array}{l}\text { http://hemeroteca.lavanguardia.com/preview/2020/03/06/pagina- } \\
\text { 21/293216776/pdf.html }\end{array}$ \\
\hline 168 & $6 / 3 / 2020$ & $\begin{array}{l}\text { http://hemeroteca.lavanguardia.com/preview/2020/03/06/pagina- } \\
\text { 22/293216643/pdf.html }\end{array}$ \\
\hline 169 & $6 / 3 / 2020$ & $\begin{array}{l}\text { http://hemeroteca.lavanguardia.com/preview/2020/03/06/pagina- } \\
\text { 22/293216643/pdf.html }\end{array}$ \\
\hline 170 & $6 / 3 / 2020$ & $\begin{array}{l}\text { http://hemeroteca.lavanguardia.com/preview/2020/03/06/pagina- } \\
\text { 24/293217063/pdf.html }\end{array}$ \\
\hline 171 & $6 / 3 / 2020$ & $\begin{array}{l}\text { http://hemeroteca.lavanguardia.com/preview/2020/03/06/pagina- } \\
\text { 24/293217063/pdf.html }\end{array}$ \\
\hline 172 & $6 / 3 / 2020$ & $\begin{array}{l}\text { http://hemeroteca.lavanguardia.com/preview/2020/03/06/pagina- } \\
\text { 41/293217207/pdf.html }\end{array}$ \\
\hline 173 & $6 / 3 / 2020$ & $\begin{array}{l}\text { http://hemeroteca.lavanguardia.com/preview/2020/03/06/pagina- } \\
\text { 46/293216980/pdf.html }\end{array}$ \\
\hline 174 & $6 / 3 / 2020$ & $\begin{array}{l}\text { http://hemeroteca.lavanguardia.com/preview/2020/03/06/pagina- } \\
\text { 46/293216980/pdf.html }\end{array}$ \\
\hline 175 & $6 / 3 / 2020$ & $\begin{array}{l}\text { http://hemeroteca.lavanguardia.com/preview/2020/03/06/pagina- } \\
\text { 47/293216976/pdf.html }\end{array}$ \\
\hline 176 & $7 / 3 / 2020$ & $\begin{array}{l}\text { http://hemeroteca.lavanguardia.com/preview/2020/03/07/pagina- } \\
\text { 1/293565006/pdf.html }\end{array}$ \\
\hline 177 & $7 / 3 / 2020$ & $\begin{array}{l}\text { http://hemeroteca.lavanguardia.com/preview/2020/03/07/pagina- } \\
\text { 23/293516911/pdf.html }\end{array}$ \\
\hline 178 & $7 / 3 / 2020$ & $\begin{array}{l}\text { http://hemeroteca.lavanguardia.com/preview/2020/03/07/pagina- } \\
\text { 24/293564940/pdf.html }\end{array}$ \\
\hline 179 & $7 / 3 / 2020$ & $\begin{array}{l}\text { http://hemeroteca.lavanguardia.com/preview/2020/03/07/pagina- } \\
\text { 25/293564932/pdf.html }\end{array}$ \\
\hline 180 & $7 / 3 / 2020$ & $\begin{array}{l}\text { http://hemeroteca.lavanguardia.com/preview/2020/03/07/pagina- } \\
\text { 25/293564932/pdf.html }\end{array}$ \\
\hline
\end{tabular}




\begin{tabular}{|c|c|c|}
\hline $\begin{array}{c}\text { Item ref. } \\
(*)\end{array}$ & Fecha & Enlace \\
\hline 181 & $7 / 3 / 2020$ & $\begin{array}{l}\text { http://hemeroteca.lavanguardia.com/preview/2020/03/07/pagina- } \\
\text { 26/293517142/pdf.html }\end{array}$ \\
\hline 182 & $7 / 3 / 2020$ & $\begin{array}{l}\text { http://hemeroteca.lavanguardia.com/preview/2020/03/07/pagina- } \\
\text { 26/293517142/pdf.html }\end{array}$ \\
\hline 183 & $7 / 3 / 2020$ & $\begin{array}{l}\text { http://hemeroteca.lavanguardia.com/preview/2020/03/07/pagina- } \\
\text { 28/293564489/pdf.html }\end{array}$ \\
\hline 184 & $7 / 3 / 2020$ & $\begin{array}{l}\text { http://hemeroteca.lavanguardia.com/preview/2020/03/07/pagina- } \\
\text { 28/293564489/pdf.html }\end{array}$ \\
\hline 185 & $7 / 3 / 2020$ & $\begin{array}{l}\text { http://hemeroteca.lavanguardia.com/preview/2020/03/07/pagina- } \\
\text { 29/293564684/pdf.html }\end{array}$ \\
\hline 186 & $7 / 3 / 2020$ & $\begin{array}{l}\text { http://hemeroteca.lavanguardia.com/preview/2020/03/07/pagina- } \\
\text { 50/293564910/pdf.html }\end{array}$ \\
\hline 187 & $7 / 3 / 2020$ & $\begin{array}{l}\text { http://hemeroteca.lavanguardia.com/preview/2020/03/07/pagina- } \\
\text { 51/293564914/pdf.html }\end{array}$ \\
\hline 188 & $8 / 3 / 2020$ & $\begin{array}{l}\text { http://hemeroteca.lavanguardia.com/preview/2020/03/08/pagina- } \\
\text { 1/293766865/pdf.html }\end{array}$ \\
\hline 189 & $8 / 3 / 2020$ & $\begin{array}{l}\text { http://hemeroteca.lavanguardia.com/preview/2020/03/08/pagina- } \\
\text { 1/293766865/pdf.html }\end{array}$ \\
\hline 190 & $8 / 3 / 2020$ & $\begin{array}{l}\text { http://hemeroteca.lavanguardia.com/preview/2020/03/08/pagina- } \\
\text { 1/293766865/pdf.html }\end{array}$ \\
\hline 191 & $8 / 3 / 2020$ & $\begin{array}{l}\text { http://hemeroteca.lavanguardia.com/preview/2020/03/08/pagina- } \\
\text { 3/293766896/pdf.html }\end{array}$ \\
\hline 192 & $8 / 3 / 2020$ & $\begin{array}{l}\text { http://hemeroteca.lavanguardia.com/preview/2020/03/08/pagina- } \\
\text { 4/293766474/pdf.html }\end{array}$ \\
\hline 193 & $8 / 3 / 2020$ & $\begin{array}{l}\text { http://hemeroteca.lavanguardia.com/preview/2020/03/08/pagina- } \\
\text { 19/293766405/pdf.html }\end{array}$ \\
\hline 194 & $8 / 3 / 2020$ & $\begin{array}{l}\text { http://hemeroteca.lavanguardia.com/preview/2020/03/08/pagina- } \\
\text { 26/293766049/pdf.html }\end{array}$ \\
\hline 195 & $8 / 3 / 2020$ & $\begin{array}{l}\text { http://hemeroteca.lavanguardia.com/preview/2020/03/08/pagina- } \\
\text { 27/293766061/pdf.html }\end{array}$ \\
\hline 196 & $8 / 3 / 2020$ & $\begin{array}{l}\text { http://hemeroteca.lavanguardia.com/preview/2020/03/08/pagina- } \\
\text { 36/293766453/pdf.html }\end{array}$ \\
\hline 197 & $8 / 3 / 2020$ & $\begin{array}{l}\text { http://hemeroteca.lavanguardia.com/preview/2020/03/08/pagina- } \\
\text { 37/293766449/pdf.html }\end{array}$ \\
\hline 198 & $8 / 3 / 2020$ & $\begin{array}{l}\text { http://hemeroteca.lavanguardia.com/preview/2020/03/08/pagina- } \\
\text { 37/293766449/pdf.html }\end{array}$ \\
\hline 199 & $8 / 3 / 2020$ & $\begin{array}{l}\text { http://hemeroteca.lavanguardia.com/preview/2020/03/08/pagina- } \\
\text { 38/293766445/pdf.html }\end{array}$ \\
\hline
\end{tabular}




\begin{tabular}{|c|c|c|}
\hline $\begin{array}{c}\text { Item ref. } \\
(*)\end{array}$ & Fecha & Enlace \\
\hline 200 & $8 / 3 / 2020$ & $\begin{array}{l}\text { http://hemeroteca.lavanguardia.com/preview/2020/03/08/pagina- } \\
\text { 38/293766445/pdf.html }\end{array}$ \\
\hline 201 & $8 / 3 / 2020$ & $\begin{array}{l}\text { http://hemeroteca.lavanguardia.com/preview/2020/03/08/pagina- } \\
\text { 38/293766445/pdf.html }\end{array}$ \\
\hline 202 & $8 / 3 / 2020$ & $\begin{array}{l}\text { http://hemeroteca.lavanguardia.com/preview/2020/03/08/pagina- } \\
\text { 38/293766445/pdf.html }\end{array}$ \\
\hline 203 & $8 / 3 / 2020$ & $\begin{array}{l}\text { http://hemeroteca.lavanguardia.com/preview/2020/03/08/pagina- } \\
\text { 38/293766445/pdf.html }\end{array}$ \\
\hline 204 & $8 / 3 / 2020$ & $\begin{array}{l}\text { http://hemeroteca.lavanguardia.com/preview/2020/03/08/pagina- } \\
\text { 38/293766445/pdf.html }\end{array}$ \\
\hline 205 & $8 / 3 / 2020$ & $\begin{array}{l}\text { http://hemeroteca.lavanguardia.com/preview/2020/03/08/pagina- } \\
\text { 38/293766445/pdf.html }\end{array}$ \\
\hline 206 & $8 / 3 / 2020$ & $\begin{array}{l}\text { http://hemeroteca.lavanguardia.com/preview/2020/03/08/pagina- } \\
\text { 39/293766421/pdf.html }\end{array}$ \\
\hline 207 & $8 / 3 / 2020$ & $\begin{array}{l}\text { http://hemeroteca.lavanguardia.com/preview/2020/03/08/pagina- } \\
\text { 64/293766542/pdf.html }\end{array}$ \\
\hline 208 & $8 / 3 / 2020$ & $\begin{array}{l}\text { http://hemeroteca.lavanguardia.com/preview/2020/03/08/pagina- } \\
\text { 68/293766104/pdf.html }\end{array}$ \\
\hline 209 & $8 / 3 / 2020$ & $\begin{array}{l}\text { http://hemeroteca.lavanguardia.com/preview/2020/03/08/pagina- } \\
\text { 69/293766112/pdf.html }\end{array}$ \\
\hline 210 & $8 / 3 / 2020$ & $\begin{array}{l}\text { http://hemeroteca.lavanguardia.com/preview/2020/03/08/pagina- } \\
\text { 70/293766029/pdf.html }\end{array}$ \\
\hline 211 & $8 / 3 / 2020$ & $\begin{array}{l}\text { http://hemeroteca.lavanguardia.com/preview/2020/03/08/pagina- } \\
\text { 70/293766029/pdf.html }\end{array}$ \\
\hline 212 & $8 / 3 / 2020$ & $\begin{array}{l}\text { http://hemeroteca.lavanguardia.com/preview/2020/03/08/pagina- } \\
\text { 72/293766033/pdf.html }\end{array}$ \\
\hline 213 & $8 / 3 / 2020$ & $\begin{array}{l}\text { http://hemeroteca.lavanguardia.com/preview/2020/03/08/pagina- } \\
\text { 73/293766221/pdf.html }\end{array}$ \\
\hline 214 & $9 / 3 / 2020$ & $\begin{array}{l}\text { http://hemeroteca.lavanguardia.com/preview/2020/03/09/pagina- } \\
\text { 1/293967845/pdf.html }\end{array}$ \\
\hline 215 & $9 / 3 / 2020$ & $\begin{array}{l}\text { http://hemeroteca.lavanguardia.com/preview/2020/03/09/pagina- } \\
\text { 2/293967621/pdf.html }\end{array}$ \\
\hline 216 & $9 / 3 / 2020$ & $\begin{array}{l}\text { http://hemeroteca.lavanguardia.com/preview/2020/03/09/pagina- } \\
\text { 3/293967804/pdf.html }\end{array}$ \\
\hline 217 & $9 / 3 / 2020$ & $\begin{array}{l}\text { http://hemeroteca.lavanguardia.com/preview/2020/03/09/pagina- } \\
\text { 4/293967796/pdf.html }\end{array}$ \\
\hline 218 & $9 / 3 / 2020$ & $\begin{array}{l}\text { http://hemeroteca.lavanguardia.com/preview/2020/03/09/pagina- } \\
\text { 6/293967611/pdf.html }\end{array}$ \\
\hline
\end{tabular}




\begin{tabular}{|c|c|c|}
\hline $\begin{array}{c}\text { Item ref. } \\
(*)\end{array}$ & Fecha & Enlace \\
\hline 219 & $9 / 3 / 2020$ & $\begin{array}{l}\text { http://hemeroteca.lavanguardia.com/preview/2020/03/09/pagina- } \\
\text { 6/293967611/pdf.html }\end{array}$ \\
\hline 220 & $9 / 3 / 2020$ & $\begin{array}{l}\text { http://hemeroteca.lavanguardia.com/preview/2020/03/09/pagina- } \\
\text { 7/293967454/pdf.html }\end{array}$ \\
\hline 221 & $9 / 3 / 2020$ & $\begin{array}{l}\text { http://hemeroteca.lavanguardia.com/preview/2020/03/09/pagina- } \\
\text { 16/293967389/pdf.html }\end{array}$ \\
\hline 222 & $9 / 3 / 2020$ & $\begin{array}{l}\text { http://hemeroteca.lavanguardia.com/preview/2020/03/09/pagina- } \\
\text { 17/293967295/pdf.html }\end{array}$ \\
\hline 223 & $9 / 3 / 2020$ & $\begin{array}{l}\text { http://hemeroteca.lavanguardia.com/preview/2020/03/09/pagina- } \\
\text { 24/293967788/pdf.html }\end{array}$ \\
\hline 224 & $9 / 3 / 2020$ & $\begin{array}{l}\text { http://hemeroteca.lavanguardia.com/preview/2020/03/09/pagina- } \\
\text { 25/293967760/pdf.html }\end{array}$ \\
\hline 225 & $9 / 3 / 2020$ & $\begin{array}{l}\text { http://hemeroteca.lavanguardia.com/preview/2020/03/09/pagina- } \\
\text { 25/293967760/pdf.html }\end{array}$ \\
\hline 226 & $9 / 3 / 2020$ & $\begin{array}{l}\text { http://hemeroteca.lavanguardia.com/preview/2020/03/09/pagina- } \\
\text { 50/293967677/pdf.html }\end{array}$ \\
\hline 227 & $9 / 3 / 2020$ & $\begin{array}{l}\text { http://hemeroteca.lavanguardia.com/preview/2020/03/09/pagina- } \\
\text { 51/293967685/pdf.html }\end{array}$ \\
\hline 228 & $9 / 3 / 2020$ & $\begin{array}{l}\text { http://hemeroteca.lavanguardia.com/preview/2020/03/09/pagina- } \\
\underline{\text { 52/293967665/pdf.html }}\end{array}$ \\
\hline 229 & $9 / 3 / 2020$ & $\begin{array}{l}\text { http://hemeroteca.lavanguardia.com/preview/2020/03/09/pagina- } \\
\text { 52/293967665/pdf.html }\end{array}$ \\
\hline 230 & $10 / 3 / 2020$ & $\begin{array}{l}\text { http://hemeroteca.lavanguardia.com/preview/2020/03/10/pagina- } \\
\text { 1/294117899/pdf.html }\end{array}$ \\
\hline 231 & $10 / 3 / 2020$ & $\begin{array}{l}\text { http://hemeroteca.lavanguardia.com/preview/2020/03/10/pagina- } \\
\text { 1/294117899/pdf.html }\end{array}$ \\
\hline 232 & $10 / 3 / 2020$ & $\begin{array}{l}\text { http://hemeroteca.lavanguardia.com/preview/2020/03/10/pagina- } \\
\text { 1/294117899/pdf.html }\end{array}$ \\
\hline 233 & $10 / 3 / 2020$ & $\begin{array}{l}\text { http://hemeroteca.lavanguardia.com/preview/2020/03/10/pagina- } \\
\text { 2/294117594/pdf.html }\end{array}$ \\
\hline 234 & $10 / 3 / 2020$ & $\begin{array}{l}\text { http://hemeroteca.lavanguardia.com/preview/2020/03/10/pagina- } \\
\text { 3/294117937/pdf.html }\end{array}$ \\
\hline 235 & $10 / 3 / 2020$ & $\begin{array}{l}\text { http://hemeroteca.lavanguardia.com/preview/2020/03/10/pagina- } \\
\text { 4/294117933/pdf.html }\end{array}$ \\
\hline 236 & $10 / 3 / 2020$ & $\begin{array}{l}\text { http://hemeroteca.lavanguardia.com/preview/2020/03/10/pagina- } \\
\text { 6/294117748/pdf.html }\end{array}$ \\
\hline 237 & $10 / 3 / 2020$ & $\begin{array}{l}\text { http://hemeroteca.lavanguardia.com/preview/2020/03/10/pagina- } \\
\text { 8/294117766/pdf.html }\end{array}$ \\
\hline
\end{tabular}




\begin{tabular}{|c|c|c|}
\hline $\begin{array}{c}\text { Item ref. } \\
(*)\end{array}$ & Fecha & Enlace \\
\hline 238 & $10 / 3 / 2020$ & $\begin{array}{l}\text { http://hemeroteca.lavanguardia.com/preview/2020/03/10/pagina- } \\
\text { 13/294117651/pdf.html }\end{array}$ \\
\hline 239 & $10 / 3 / 2020$ & $\begin{array}{l}\text { http://hemeroteca.lavanguardia.com/preview/2020/03/10/pagina- } \\
\text { 20/294117398/pdf.html }\end{array}$ \\
\hline 240 & $10 / 3 / 2020$ & $\begin{array}{l}\text { http://hemeroteca.lavanguardia.com/preview/2020/03/10/pagina- } \\
\text { 20/294117398/pdf.html }\end{array}$ \\
\hline 241 & $10 / 3 / 2020$ & $\begin{array}{l}\text { http://hemeroteca.lavanguardia.com/preview/2020/03/10/pagina- } \\
\text { 24/294117822/pdf.html }\end{array}$ \\
\hline 242 & $10 / 3 / 2020$ & $\begin{array}{l}\text { http://hemeroteca.lavanguardia.com/preview/2020/03/10/pagina- } \\
\text { 25/294117818/pdf.html }\end{array}$ \\
\hline 243 & $10 / 3 / 2020$ & $\begin{array}{l}\text { http://hemeroteca.lavanguardia.com/preview/2020/03/10/pagina- } \\
\text { 25/294117818/pdf.html }\end{array}$ \\
\hline 244 & $10 / 3 / 2020$ & $\begin{array}{l}\text { http://hemeroteca.lavanguardia.com/preview/2020/03/10/pagina- } \\
\text { 26/294117612/pdf.html }\end{array}$ \\
\hline 245 & $10 / 3 / 2020$ & $\begin{array}{l}\text { http://hemeroteca.lavanguardia.com/preview/2020/03/10/pagina- } \\
\text { 26/294117612/pdf.html }\end{array}$ \\
\hline 246 & $10 / 3 / 2020$ & $\begin{array}{l}\text { http://hemeroteca.lavanguardia.com/preview/2020/03/10/pagina- } \\
\text { 27/294117517/pdf.html }\end{array}$ \\
\hline 247 & $10 / 3 / 2020$ & $\begin{array}{l}\text { http://hemeroteca.lavanguardia.com/preview/2020/03/10/pagina- } \\
27 / 294117517 / \text { pdf.html }\end{array}$ \\
\hline 248 & $10 / 3 / 2020$ & $\begin{array}{l}\text { http://hemeroteca.lavanguardia.com/preview/2020/03/10/pagina- } \\
\text { 44/294117734/pdf.html }\end{array}$ \\
\hline 249 & $10 / 3 / 2020$ & $\begin{array}{l}\text { http://hemeroteca.lavanguardia.com/preview/2020/03/10/pagina- } \\
\text { 45/294117730/pdf.html }\end{array}$ \\
\hline 250 & $10 / 3 / 2020$ & $\begin{array}{l}\text { http://hemeroteca.lavanguardia.com/preview/2020/03/10/pagina- } \\
\text { 46/294117500/pdf.html }\end{array}$ \\
\hline 251 & $10 / 3 / 2020$ & $\begin{array}{l}\text { http://hemeroteca.lavanguardia.com/preview/2020/03/10/pagina- } \\
\text { 47/294117496/pdf.html }\end{array}$ \\
\hline 252 & $10 / 3 / 2020$ & $\begin{array}{l}\text { http://hemeroteca.lavanguardia.com/preview/2020/03/10/pagina- } \\
\text { 48/294118072/pdf.html }\end{array}$ \\
\hline 253 & $10 / 3 / 2020$ & $\begin{array}{l}\text { http://hemeroteca.lavanguardia.com/preview/2020/03/10/pagina- } \\
\text { 48/294118072/pdf.html }\end{array}$ \\
\hline 254 & $10 / 3 / 2020$ & $\begin{array}{l}\text { http://hemeroteca.lavanguardia.com/preview/2020/03/10/pagina- } \\
\text { 49/294117863/pdf.html }\end{array}$ \\
\hline 255 & $11 / 3 / 2020$ & $\begin{array}{l}\text { http://hemeroteca.lavanguardia.com/preview/2020/03/11/pagina- } \\
\text { 1/294367408/pdf.html }\end{array}$ \\
\hline 256 & $11 / 3 / 2020$ & $\begin{array}{l}\text { http://hemeroteca.lavanguardia.com/preview/2020/03/11/pagina- } \\
\text { 1/294367408/pdf.html }\end{array}$ \\
\hline
\end{tabular}




\begin{tabular}{|c|c|c|}
\hline $\begin{array}{c}\text { Item ref. } \\
(*)\end{array}$ & Fecha & Enlace \\
\hline 257 & $11 / 3 / 2020$ & $\begin{array}{l}\text { http://hemeroteca.lavanguardia.com/preview/2020/03/11/pagina- } \\
\text { 1/294367408/pdf.html }\end{array}$ \\
\hline 258 & $11 / 3 / 2020$ & $\begin{array}{l}\text { http://hemeroteca.lavanguardia.com/preview/2020/03/11/pagina- } \\
\text { 1/294367408/pdf.html }\end{array}$ \\
\hline 259 & $11 / 3 / 2020$ & $\begin{array}{l}\text { http://hemeroteca.lavanguardia.com/preview/2020/03/11/pagina- } \\
\text { 1/294367408/pdf.html }\end{array}$ \\
\hline 260 & $11 / 3 / 2020$ & $\begin{array}{l}\text { http://hemeroteca.lavanguardia.com/preview/2020/03/11/pagina- } \\
\text { 2/294367326/pdf.html }\end{array}$ \\
\hline 261 & $11 / 3 / 2020$ & $\begin{array}{l}\text { http://hemeroteca.lavanguardia.com/preview/2020/03/11/pagina- } \\
\text { 3/294367118/pdf.html }\end{array}$ \\
\hline 262 & $11 / 3 / 2020$ & $\begin{array}{l}\text { http://hemeroteca.lavanguardia.com/preview/2020/03/11/pagina- } \\
\text { 4/294367136/pdf.html }\end{array}$ \\
\hline 263 & $11 / 3 / 2020$ & $\begin{array}{l}\text { http://hemeroteca.lavanguardia.com/preview/2020/03/11/pagina- } \\
\text { 6/294367339/pdf.html }\end{array}$ \\
\hline 264 & $11 / 3 / 2020$ & $\begin{array}{l}\text { http://hemeroteca.lavanguardia.com/preview/2020/03/11/pagina- } \\
\text { 6/294367339/pdf.html }\end{array}$ \\
\hline 265 & $11 / 3 / 2020$ & $\begin{array}{l}\text { http://hemeroteca.lavanguardia.com/preview/2020/03/11/pagina- } \\
\text { 8/294367358/pdf.html }\end{array}$ \\
\hline 266 & $11 / 3 / 2020$ & $\begin{array}{l}\text { http://hemeroteca.lavanguardia.com/preview/2020/03/11/pagina- } \\
\text { 12/294367735/pdf.html }\end{array}$ \\
\hline 267 & $11 / 3 / 2020$ & $\begin{array}{l}\text { http://hemeroteca.lavanguardia.com/preview/2020/03/11/pagina- } \\
\text { 13/294367740/pdf.html }\end{array}$ \\
\hline 268 & $11 / 3 / 2020$ & $\begin{array}{l}\text { http://hemeroteca.lavanguardia.com/preview/2020/03/11/pagina- } \\
\text { 14/294367469/pdf.html }\end{array}$ \\
\hline 269 & $11 / 3 / 2020$ & $\begin{array}{l}\text { http://hemeroteca.lavanguardia.com/preview/2020/03/11/pagina- } \\
\text { 14/294367469/pdf.html }\end{array}$ \\
\hline 270 & $11 / 3 / 2020$ & $\begin{array}{l}\text { http://hemeroteca.lavanguardia.com/preview/2020/03/11/pagina- } \\
\text { 18/294367051/pdf.html }\end{array}$ \\
\hline 271 & $11 / 3 / 2020$ & $\begin{array}{l}\text { http://hemeroteca.lavanguardia.com/preview/2020/03/11/pagina- } \\
\text { 19/294366908/pdf.html }\end{array}$ \\
\hline 272 & $11 / 3 / 2020$ & $\begin{array}{l}\text { http://hemeroteca.lavanguardia.com/preview/2020/03/11/pagina- } \\
\text { 20/294367530/pdf.html }\end{array}$ \\
\hline 273 & $11 / 3 / 2020$ & $\begin{array}{l}\text { http://hemeroteca.lavanguardia.com/preview/2020/03/11/pagina- } \\
\text { 22/294367424/pdf.html }\end{array}$ \\
\hline 274 & $11 / 3 / 2020$ & $\begin{array}{l}\text { http://hemeroteca.lavanguardia.com/preview/2020/03/11/pagina- } \\
\text { 23/294367428/pdf.html }\end{array}$ \\
\hline 275 & $11 / 3 / 2020$ & $\begin{array}{l}\text { http://hemeroteca.lavanguardia.com/preview/2020/03/11/pagina- } \\
\text { 23/294367428/pdf.html }\end{array}$ \\
\hline
\end{tabular}




\begin{tabular}{|c|c|c|}
\hline $\begin{array}{l}\text { Item ref. } \\
(*)\end{array}$ & Fecha & Enlace \\
\hline 276 & $11 / 3 / 2020$ & $\begin{array}{l}\text { http://hemeroteca.lavanguardia.com/preview/2020/03/11/pagina- } \\
\text { 24/294367362/pdf.html }\end{array}$ \\
\hline 277 & $11 / 3 / 2020$ & $\begin{array}{l}\text { http://hemeroteca.lavanguardia.com/preview/2020/03/11/pagina- } \\
\text { 24/294367362/pdf.html }\end{array}$ \\
\hline 278 & $11 / 3 / 2020$ & $\begin{array}{l}\text { http://hemeroteca.lavanguardia.com/preview/2020/03/11/pagina- } \\
\text { 25/294367146/pdf.html }\end{array}$ \\
\hline 279 & $11 / 3 / 2020$ & $\begin{array}{l}\text { http://hemeroteca.lavanguardia.com/preview/2020/03/11/pagina- } \\
\text { 25/294367146/pdf.html }\end{array}$ \\
\hline 280 & $11 / 3 / 2020$ & $\begin{array}{l}\text { http://hemeroteca.lavanguardia.com/preview/2020/03/11/pagina- } \\
\text { 26/294366920/pdf.html }\end{array}$ \\
\hline 281 & $11 / 3 / 2020$ & $\begin{array}{l}\text { http://hemeroteca.lavanguardia.com/preview/2020/03/11/pagina- } \\
\text { 26/294366920/pdf.html }\end{array}$ \\
\hline 282 & $11 / 3 / 2020$ & $\begin{array}{l}\text { http://hemeroteca.lavanguardia.com/preview/2020/03/11/pagina- } \\
\text { 30/294367518/pdf.html }\end{array}$ \\
\hline 283 & $11 / 3 / 2020$ & $\begin{array}{l}\text { http://hemeroteca.lavanguardia.com/preview/2020/03/11/pagina- } \\
\text { 38/294367250/pdf.html }\end{array}$ \\
\hline 284 & $11 / 3 / 2020$ & $\begin{array}{l}\text { http://hemeroteca.lavanguardia.com/preview/2020/03/11/pagina- } \\
\text { 44/294367510/pdf.html }\end{array}$ \\
\hline 285 & $11 / 3 / 2020$ & $\begin{array}{l}\text { http://hemeroteca.lavanguardia.com/preview/2020/03/11/pagina- } \\
\text { 44/294367510/pdf.html }\end{array}$ \\
\hline 286 & $11 / 3 / 2020$ & $\begin{array}{l}\text { http://hemeroteca.lavanguardia.com/preview/2020/03/11/pagina- } \\
\text { 45/294367506/pdf.html }\end{array}$ \\
\hline 287 & $11 / 3 / 2020$ & $\begin{array}{l}\text { http://hemeroteca.lavanguardia.com/preview/2020/03/11/pagina- } \\
\text { 46/294367502/pdf.html }\end{array}$ \\
\hline 288 & $11 / 3 / 2020$ & $\begin{array}{l}\text { http://hemeroteca.lavanguardia.com/preview/2020/03/11/pagina- } \\
\text { 46/294367502/pdf.html }\end{array}$ \\
\hline 289 & $11 / 3 / 2020$ & $\begin{array}{l}\text { http://hemeroteca.lavanguardia.com/preview/2020/03/11/pagina- } \\
\text { 47/294367549/pdf.html }\end{array}$ \\
\hline 290 & $11 / 3 / 2020$ & $\begin{array}{l}\text { http://hemeroteca.lavanguardia.com/preview/2020/03/11/pagina- } \\
\text { 47/294367549/pdf.html }\end{array}$ \\
\hline 291 & $12 / 3 / 2020$ & $\begin{array}{l}\text { http://hemeroteca.lavanguardia.com/preview/2020/03/12/pagina- } \\
\text { 1/294467325/pdf.html }\end{array}$ \\
\hline 292 & $12 / 3 / 2020$ & $\begin{array}{l}\text { http://hemeroteca.lavanguardia.com/preview/2020/03/12/pagina- } \\
1 / 294467325 / \text { pdf.html }\end{array}$ \\
\hline 293 & $12 / 3 / 2020$ & $\begin{array}{l}\text { http://hemeroteca.lavanguardia.com/preview/2020/03/12/pagina- } \\
\text { 2/294467311/pdf.html }\end{array}$ \\
\hline 294 & $12 / 3 / 2020$ & $\begin{array}{l}\text { http://hemeroteca.lavanguardia.com/preview/2020/03/12/pagina- } \\
\text { 3/294664473/pdf.html }\end{array}$ \\
\hline
\end{tabular}




\begin{tabular}{|c|c|c|}
\hline $\begin{array}{c}\text { Item ref. } \\
(*)\end{array}$ & Fecha & Enlace \\
\hline 295 & $12 / 3 / 2020$ & $\begin{array}{l}\text { http://hemeroteca.lavanguardia.com/preview/2020/03/12/pagina- } \\
\text { 3/294664473/pdf.html }\end{array}$ \\
\hline 296 & $12 / 3 / 2020$ & $\begin{array}{l}\text { http://hemeroteca.lavanguardia.com/preview/2020/03/12/pagina- } \\
\text { 4/294664463/pdf.html }\end{array}$ \\
\hline 297 & $12 / 3 / 2020$ & $\begin{array}{l}\text { http://hemeroteca.lavanguardia.com/preview/2020/03/12/pagina- } \\
\text { 5/294467297/pdf.html }\end{array}$ \\
\hline 298 & $12 / 3 / 2020$ & $\begin{array}{l}\text { http://hemeroteca.lavanguardia.com/preview/2020/03/12/pagina- } \\
\text { 5/294467297/pdf.html }\end{array}$ \\
\hline 299 & $12 / 3 / 2020$ & $\begin{array}{l}\text { http://hemeroteca.lavanguardia.com/preview/2020/03/12/pagina- } \\
\text { 12/294467429/pdf.html }\end{array}$ \\
\hline 300 & $12 / 3 / 2020$ & $\begin{array}{l}\text { http://hemeroteca.lavanguardia.com/preview/2020/03/12/pagina- } \\
\text { 13/294467441/pdf.html }\end{array}$ \\
\hline 301 & $12 / 3 / 2020$ & $\begin{array}{l}\text { http://hemeroteca.lavanguardia.com/preview/2020/03/12/pagina- } \\
\text { 14/294467289/pdf.html }\end{array}$ \\
\hline 302 & $12 / 3 / 2020$ & $\begin{array}{l}\text { http://hemeroteca.lavanguardia.com/preview/2020/03/12/pagina- } \\
\text { 15/294467147/pdf.html }\end{array}$ \\
\hline 303 & $12 / 3 / 2020$ & $\begin{array}{l}\text { http://hemeroteca.lavanguardia.com/preview/2020/03/12/pagina- } \\
\text { 20/294467028/pdf.html }\end{array}$ \\
\hline 304 & $12 / 3 / 2020$ & $\begin{array}{l}\text { http://hemeroteca.lavanguardia.com/preview/2020/03/12/pagina- } \\
\text { 21/294467213/pdf.html }\end{array}$ \\
\hline 305 & $12 / 3 / 2020$ & $\begin{array}{l}\text { http://hemeroteca.lavanguardia.com/preview/2020/03/12/pagina- } \\
\text { 24/294467267/pdf.html }\end{array}$ \\
\hline 306 & $12 / 3 / 2020$ & $\begin{array}{l}\text { http://hemeroteca.lavanguardia.com/preview/2020/03/12/pagina- } \\
\text { 25/294467285/pdf.html }\end{array}$ \\
\hline 307 & $12 / 3 / 2020$ & $\begin{array}{l}\text { http://hemeroteca.lavanguardia.com/preview/2020/03/12/pagina- } \\
\text { 25/294467285/pdf.html }\end{array}$ \\
\hline 308 & $12 / 3 / 2020$ & $\begin{array}{l}\text { http://hemeroteca.lavanguardia.com/preview/2020/03/12/pagina- } \\
\text { 26/294467089/pdf.html }\end{array}$ \\
\hline 309 & $12 / 3 / 2020$ & $\begin{array}{l}\text { http://hemeroteca.lavanguardia.com/preview/2020/03/12/pagina- } \\
\text { 26/294467089/pdf.html }\end{array}$ \\
\hline 310 & $12 / 3 / 2020$ & $\begin{array}{l}\text { http://hemeroteca.lavanguardia.com/preview/2020/03/12/pagina- } \\
\text { 27/294467151/pdf.html }\end{array}$ \\
\hline 311 & $12 / 3 / 2020$ & $\begin{array}{l}\text { http://hemeroteca.lavanguardia.com/preview/2020/03/12/pagina- } \\
\text { 34/294467476/pdf.html }\end{array}$ \\
\hline 312 & $12 / 3 / 2020$ & $\begin{array}{l}\text { http://hemeroteca.lavanguardia.com/preview/2020/03/12/pagina- } \\
\text { 35/294467480/pdf.html }\end{array}$ \\
\hline 313 & $12 / 3 / 2020$ & $\begin{array}{l}\text { http://hemeroteca.lavanguardia.com/preview/2020/03/12/pagina- } \\
\text { 60/294467232/pdf.html }\end{array}$ \\
\hline
\end{tabular}




\begin{tabular}{|c|c|c|}
\hline $\begin{array}{c}\text { Item ref. } \\
(*)\end{array}$ & Fecha & Enlace \\
\hline 314 & $12 / 3 / 2020$ & $\begin{array}{l}\text { http://hemeroteca.lavanguardia.com/preview/2020/03/12/pagina- } \\
\text { 61/294467228/pdf.html }\end{array}$ \\
\hline 315 & $12 / 3 / 2020$ & $\begin{array}{l}\text { http://hemeroteca.lavanguardia.com/preview/2020/03/12/pagina- } \\
\text { 62/294467370/pdf.html }\end{array}$ \\
\hline 316 & $12 / 3 / 2020$ & $\begin{array}{l}\text { http://hemeroteca.lavanguardia.com/preview/2020/03/12/pagina- } \\
\text { 62/294467370/pdf.html }\end{array}$ \\
\hline 317 & $12 / 3 / 2020$ & $\begin{array}{l}\text { http://hemeroteca.lavanguardia.com/preview/2020/03/12/pagina- } \\
\text { 63/294467240/pdf.html }\end{array}$ \\
\hline 318 & $12 / 3 / 2020$ & $\begin{array}{l}\text { http://hemeroteca.lavanguardia.com/preview/2020/03/12/pagina- } \\
\text { 63/294467240/pdf.html }\end{array}$ \\
\hline 319 & $12 / 3 / 2020$ & $\begin{array}{l}\text { http://hemeroteca.lavanguardia.com/preview/2020/03/12/pagina- } \\
\text { 64/294467127/pdf.html }\end{array}$ \\
\hline 320 & $12 / 3 / 2020$ & $\begin{array}{l}\text { http://hemeroteca.lavanguardia.com/preview/2020/03/12/pagina- } \\
\text { 65/294467135/pdf.html }\end{array}$ \\
\hline 321 & $13 / 3 / 2020$ & $\begin{array}{l}\text { http://hemeroteca.lavanguardia.com/preview/2020/03/13/pagina- } \\
\text { 1/294766797/pdf.html }\end{array}$ \\
\hline 322 & $13 / 3 / 2020$ & $\begin{array}{l}\text { http://hemeroteca.lavanguardia.com/preview/2020/03/13/pagina- } \\
\text { 1/294766797/pdf.html }\end{array}$ \\
\hline 323 & $13 / 3 / 2020$ & $\begin{array}{l}\text { http://hemeroteca.lavanguardia.com/preview/2020/03/13/pagina- } \\
\text { 2/294766785/pdf.html }\end{array}$ \\
\hline 324 & $13 / 3 / 2020$ & $\begin{array}{l}\text { http://hemeroteca.lavanguardia.com/preview/2020/03/13/pagina- } \\
\text { 3/294766955/pdf.html }\end{array}$ \\
\hline 325 & $13 / 3 / 2020$ & $\begin{array}{l}\text { http://hemeroteca.lavanguardia.com/preview/2020/03/13/pagina- } \\
\text { 4/294766603/pdf.html }\end{array}$ \\
\hline 326 & $13 / 3 / 2020$ & $\begin{array}{l}\text { http://hemeroteca.lavanguardia.com/preview/2020/03/13/pagina- } \\
\text { 6/294766645/pdf.html }\end{array}$ \\
\hline 327 & $13 / 3 / 2020$ & $\begin{array}{l}\text { http://hemeroteca.lavanguardia.com/preview/2020/03/13/pagina- } \\
\text { 6/294766645/pdf.html }\end{array}$ \\
\hline 328 & $13 / 3 / 2020$ & $\begin{array}{l}\text { http://hemeroteca.lavanguardia.com/preview/2020/03/13/pagina- } \\
\text { 8/294766461/pdf.html }\end{array}$ \\
\hline 329 & $13 / 3 / 2020$ & $\begin{array}{l}\text { http://hemeroteca.lavanguardia.com/preview/2020/03/13/pagina- } \\
\text { 13/294767025/pdf.html }\end{array}$ \\
\hline 330 & $13 / 3 / 2020$ & $\begin{array}{l}\text { http://hemeroteca.lavanguardia.com/preview/2020/03/13/pagina- } \\
\text { 14/294766820/pdf.html }\end{array}$ \\
\hline 331 & $13 / 3 / 2020$ & $\begin{array}{l}\text { http://hemeroteca.lavanguardia.com/preview/2020/03/13/pagina- } \\
\text { 15/294766847/pdf.html }\end{array}$ \\
\hline 332 & $13 / 3 / 2020$ & $\begin{array}{l}\text { http://hemeroteca.lavanguardia.com/preview/2020/03/13/pagina- } \\
\text { 16/294766861/pdf.html }\end{array}$ \\
\hline
\end{tabular}




\begin{tabular}{|c|c|c|}
\hline $\begin{array}{c}\text { Item ref. } \\
(*)\end{array}$ & Fecha & Enlace \\
\hline 333 & $13 / 3 / 2020$ & $\begin{array}{l}\text { http://hemeroteca.lavanguardia.com/preview/2020/03/13/pagina- } \\
\text { 18/294766564/pdf.html }\end{array}$ \\
\hline 334 & $13 / 3 / 2020$ & $\begin{array}{l}\text { http://hemeroteca.lavanguardia.com/preview/2020/03/13/pagina- } \\
\text { 18/294766564/pdf.html }\end{array}$ \\
\hline 335 & $13 / 3 / 2020$ & $\begin{array}{l}\text { http://hemeroteca.lavanguardia.com/preview/2020/03/13/pagina- } \\
\text { 20/294766327/pdf.html }\end{array}$ \\
\hline 336 & $13 / 3 / 2020$ & $\begin{array}{l}\text { http://hemeroteca.lavanguardia.com/preview/2020/03/13/pagina- } \\
\text { 21/294766323/pdf.html }\end{array}$ \\
\hline 337 & $13 / 3 / 2020$ & $\begin{array}{l}\text { http://hemeroteca.lavanguardia.com/preview/2020/03/13/pagina- } \\
\text { 22/294766809/pdf.html }\end{array}$ \\
\hline 338 & $13 / 3 / 2020$ & $\begin{array}{l}\text { http://hemeroteca.lavanguardia.com/preview/2020/03/13/pagina- } \\
\text { 23/294766832/pdf.html }\end{array}$ \\
\hline 339 & $13 / 3 / 2020$ & $\begin{array}{l}\text { http://hemeroteca.lavanguardia.com/preview/2020/03/13/pagina- } \\
\text { 23/294766832/pdf.html }\end{array}$ \\
\hline 340 & $13 / 3 / 2020$ & $\begin{array}{l}\text { http://hemeroteca.lavanguardia.com/preview/2020/03/13/pagina- } \\
\text { 24/294766816/pdf.html }\end{array}$ \\
\hline 341 & $13 / 3 / 2020$ & $\begin{array}{l}\text { http://hemeroteca.lavanguardia.com/preview/2020/03/13/pagina- } \\
\text { 25/294766987/pdf.html }\end{array}$ \\
\hline 342 & $13 / 3 / 2020$ & $\begin{array}{l}\text { http://hemeroteca.lavanguardia.com/preview/2020/03/13/pagina- } \\
\text { 25/294766987/pdf.html }\end{array}$ \\
\hline 343 & $13 / 3 / 2020$ & $\begin{array}{l}\text { http://hemeroteca.lavanguardia.com/preview/2020/03/13/pagina- } \\
\text { 26/294766525/pdf.html }\end{array}$ \\
\hline 344 & $13 / 3 / 2020$ & $\begin{array}{l}\text { http://hemeroteca.lavanguardia.com/preview/2020/03/13/pagina- } \\
\text { 26/294766525/pdf.html }\end{array}$ \\
\hline 345 & $13 / 3 / 2020$ & $\begin{array}{l}\text { http://hemeroteca.lavanguardia.com/preview/2020/03/13/pagina- } \\
\text { 27/294766939/pdf.html }\end{array}$ \\
\hline 346 & $13 / 3 / 2020$ & $\begin{array}{l}\text { http://hemeroteca.lavanguardia.com/preview/2020/03/13/pagina- } \\
\text { 44/294767143/pdf.html }\end{array}$ \\
\hline 347 & $13 / 3 / 2020$ & $\begin{array}{l}\text { http://hemeroteca.lavanguardia.com/preview/2020/03/13/pagina- } \\
\text { 45/294767148/pdf.html }\end{array}$ \\
\hline 348 & $13 / 3 / 2020$ & $\begin{array}{l}\text { http://hemeroteca.lavanguardia.com/preview/2020/03/13/pagina- } \\
\text { 46/294766741/pdf.html }\end{array}$ \\
\hline 349 & $13 / 3 / 2020$ & $\begin{array}{l}\text { http://hemeroteca.lavanguardia.com/preview/2020/03/13/pagina- } \\
\text { 46/294766741/pdf.html }\end{array}$ \\
\hline 350 & $13 / 3 / 2020$ & $\begin{array}{l}\text { http://hemeroteca.lavanguardia.com/preview/2020/03/13/pagina- } \\
\text { 47/294766715/pdf.html }\end{array}$ \\
\hline 351 & $13 / 3 / 2020$ & $\begin{array}{l}\text { http://hemeroteca.lavanguardia.com/preview/2020/03/13/pagina- } \\
\text { 48/294766649/pdf.html }\end{array}$ \\
\hline
\end{tabular}




\begin{tabular}{|c|c|c|}
\hline $\begin{array}{c}\text { Item ref. } \\
(*)\end{array}$ & Fecha & Enlace \\
\hline 352 & $13 / 3 / 2020$ & $\begin{array}{l}\text { http://hemeroteca.lavanguardia.com/preview/2020/03/13/pagina- } \\
\text { 49/294766927/pdf.html }\end{array}$ \\
\hline 353 & $13 / 3 / 2020$ & $\begin{array}{l}\text { http://hemeroteca.lavanguardia.com/preview/2020/03/13/pagina- } \\
\text { 51/294766611/pdf.html }\end{array}$ \\
\hline 354 & $14 / 3 / 2020$ & $\begin{array}{l}\text { http://hemeroteca.lavanguardia.com/preview/2020/03/14/pagina- } \\
\text { 1/294917375/pdf.html }\end{array}$ \\
\hline 355 & $14 / 3 / 2020$ & $\begin{array}{l}\text { http://hemeroteca.lavanguardia.com/preview/2020/03/14/pagina- } \\
\text { 1/294917375/pdf.html }\end{array}$ \\
\hline 356 & $14 / 3 / 2020$ & $\begin{array}{l}\text { http://hemeroteca.lavanguardia.com/preview/2020/03/14/pagina- } \\
\text { 2/294917045/pdf.html }\end{array}$ \\
\hline 357 & $14 / 3 / 2020$ & $\begin{array}{l}\text { http://hemeroteca.lavanguardia.com/preview/2020/03/14/pagina- } \\
\text { 3/294917419/pdf.html }\end{array}$ \\
\hline 358 & $14 / 3 / 2020$ & $\begin{array}{l}\text { http://hemeroteca.lavanguardia.com/preview/2020/03/14/pagina- } \\
\text { 4/294917414/pdf.html }\end{array}$ \\
\hline 359 & $14 / 3 / 2020$ & $\begin{array}{l}\text { http://hemeroteca.lavanguardia.com/preview/2020/03/14/pagina- } \\
\text { 5/294916925/pdf.html }\end{array}$ \\
\hline 360 & $14 / 3 / 2020$ & $\begin{array}{l}\text { http://hemeroteca.lavanguardia.com/preview/2020/03/14/pagina- } \\
7 / 294916835 / \text { pdf.html }\end{array}$ \\
\hline 361 & $14 / 3 / 2020$ & $\begin{array}{l}\text { http://hemeroteca.lavanguardia.com/preview/2020/03/14/pagina- } \\
\text { 10/294917202/pdf.html }\end{array}$ \\
\hline 362 & $14 / 3 / 2020$ & $\begin{array}{l}\text { http://hemeroteca.lavanguardia.com/preview/2020/03/14/pagina- } \\
\text { 11/294917206/pdf.html }\end{array}$ \\
\hline 363 & $14 / 3 / 2020$ & $\begin{array}{l}\text { http://hemeroteca.lavanguardia.com/preview/2020/03/14/pagina- } \\
\text { 13/294917222/pdf.html }\end{array}$ \\
\hline 364 & $14 / 3 / 2020$ & $\begin{array}{l}\text { http://hemeroteca.lavanguardia.com/preview/2020/03/14/pagina- } \\
\text { 13/294917222/pdf.html }\end{array}$ \\
\hline 365 & $14 / 3 / 2020$ & $\begin{array}{l}\text { http://hemeroteca.lavanguardia.com/preview/2020/03/14/pagina- } \\
\text { 16/294916780/pdf.html }\end{array}$ \\
\hline 366 & $14 / 3 / 2020$ & $\begin{array}{l}\text { http://hemeroteca.lavanguardia.com/preview/2020/03/14/pagina- } \\
\text { 17/294916784/pdf.html }\end{array}$ \\
\hline 367 & $14 / 3 / 2020$ & $\begin{array}{l}\text { http://hemeroteca.lavanguardia.com/preview/2020/03/14/pagina- } \\
\text { 21/294916708/pdf.html }\end{array}$ \\
\hline 368 & $14 / 3 / 2020$ & $\begin{array}{l}\text { http://hemeroteca.lavanguardia.com/preview/2020/03/14/pagina- } \\
\text { 22/294917239/pdf.html }\end{array}$ \\
\hline 369 & $14 / 3 / 2020$ & $\begin{array}{l}\text { http://hemeroteca.lavanguardia.com/preview/2020/03/14/pagina- } \\
\text { 23/294917243/pdf.html }\end{array}$ \\
\hline 370 & $14 / 3 / 2020$ & $\begin{array}{l}\text { http://hemeroteca.lavanguardia.com/preview/2020/03/14/pagina- } \\
\text { 24/294917154/pdf.html }\end{array}$ \\
\hline
\end{tabular}




\begin{tabular}{|c|c|c|}
\hline $\begin{array}{c}\text { Item ref. } \\
(*)\end{array}$ & Fecha & Enlace \\
\hline 371 & $14 / 3 / 2020$ & $\begin{array}{l}\text { http://hemeroteca.lavanguardia.com/preview/2020/03/14/pagina- } \\
\text { 24/294917154/pdf.html }\end{array}$ \\
\hline 372 & $14 / 3 / 2020$ & $\begin{array}{l}\text { http://hemeroteca.lavanguardia.com/preview/2020/03/14/pagina- } \\
\text { 25/294917393/pdf.html }\end{array}$ \\
\hline 373 & $14 / 3 / 2020$ & $\begin{array}{l}\text { http://hemeroteca.lavanguardia.com/preview/2020/03/14/pagina- } \\
\text { 26/294917037/pdf.html }\end{array}$ \\
\hline 374 & $14 / 3 / 2020$ & $\begin{array}{l}\text { http://hemeroteca.lavanguardia.com/preview/2020/03/14/pagina- } \\
\text { 27/294917029/pdf.html }\end{array}$ \\
\hline 375 & $14 / 3 / 2020$ & $\begin{array}{l}\text { http://hemeroteca.lavanguardia.com/preview/2020/03/14/pagina- } \\
\text { 28/294917150/pdf.html }\end{array}$ \\
\hline 376 & $14 / 3 / 2020$ & $\begin{array}{l}\text { http://hemeroteca.lavanguardia.com/preview/2020/03/14/pagina- } \\
\text { 28/294917150/pdf.html }\end{array}$ \\
\hline 377 & $14 / 3 / 2020$ & $\begin{array}{l}\text { http://hemeroteca.lavanguardia.com/preview/2020/03/14/pagina- } \\
\text { 29/294916942/pdf.html }\end{array}$ \\
\hline 378 & $14 / 3 / 2020$ & $\begin{array}{l}\text { http://hemeroteca.lavanguardia.com/preview/2020/03/14/pagina- } \\
\text { 29/294916942/pdf.html }\end{array}$ \\
\hline 379 & $14 / 3 / 2020$ & $\begin{array}{l}\text { http://hemeroteca.lavanguardia.com/preview/2020/03/14/pagina- } \\
\text { 30/294916808/pdf.html }\end{array}$ \\
\hline 380 & $14 / 3 / 2020$ & $\begin{array}{l}\text { http://hemeroteca.lavanguardia.com/preview/2020/03/14/pagina- } \\
\text { 30/294916808/pdf.html }\end{array}$ \\
\hline 381 & $14 / 3 / 2020$ & $\begin{array}{l}\text { http://hemeroteca.lavanguardia.com/preview/2020/03/14/pagina- } \\
\text { 34/294917099/pdf.html }\end{array}$ \\
\hline 382 & $14 / 3 / 2020$ & $\begin{array}{l}\text { http://hemeroteca.lavanguardia.com/preview/2020/03/14/pagina- } \\
\text { 43/294916819/pdf.html }\end{array}$ \\
\hline 383 & $14 / 3 / 2020$ & $\begin{array}{l}\text { http://hemeroteca.lavanguardia.com/preview/2020/03/14/pagina- } \\
\text { 48/294917127/pdf.html }\end{array}$ \\
\hline 384 & $14 / 3 / 2020$ & $\begin{array}{l}\text { http://hemeroteca.lavanguardia.com/preview/2020/03/14/pagina- } \\
\text { 49/294917131/pdf.html }\end{array}$ \\
\hline 385 & $14 / 3 / 2020$ & $\begin{array}{l}\text { http://hemeroteca.lavanguardia.com/preview/2020/03/14/pagina- } \\
\text { 50/294917017/pdf.html }\end{array}$ \\
\hline 386 & $14 / 3 / 2020$ & $\begin{array}{l}\text { http://hemeroteca.lavanguardia.com/preview/2020/03/14/pagina- } \\
\text { 51/294917002/pdf.html }\end{array}$ \\
\hline 387 & $14 / 3 / 2020$ & $\begin{array}{l}\text { http://hemeroteca.lavanguardia.com/preview/2020/03/14/pagina- } \\
\text { 51/294917002/pdf.html }\end{array}$ \\
\hline 388 & $14 / 3 / 2020$ & $\begin{array}{l}\text { http://hemeroteca.lavanguardia.com/preview/2020/03/14/pagina- } \\
\text { 56/294917178/pdf.html }\end{array}$ \\
\hline 389 & $15 / 3 / 2020$ & $\begin{array}{l}\text { http://hemeroteca.lavanguardia.com/preview/2020/03/15/pagina- } \\
\text { 1/295116920/pdf.html }\end{array}$ \\
\hline
\end{tabular}




\begin{tabular}{|c|c|c|}
\hline $\begin{array}{c}\text { Item ref. } \\
(*)\end{array}$ & Fecha & Enlace \\
\hline 390 & $15 / 3 / 2020$ & $\begin{array}{l}\text { http://hemeroteca.lavanguardia.com/preview/2020/03/15/pagina- } \\
\text { 1/295116920/pdf.html }\end{array}$ \\
\hline 391 & $15 / 3 / 2020$ & $\begin{array}{l}\text { http://hemeroteca.lavanguardia.com/preview/2020/03/15/pagina- } \\
\text { 2/295116652/pdf.html }\end{array}$ \\
\hline 392 & $15 / 3 / 2020$ & $\begin{array}{l}\text { http://hemeroteca.lavanguardia.com/preview/2020/03/15/pagina- } \\
\text { 3/295116713/pdf.html }\end{array}$ \\
\hline 393 & $15 / 3 / 2020$ & $\begin{array}{l}\text { http://hemeroteca.lavanguardia.com/preview/2020/03/15/pagina- } \\
\text { 8/295116593/pdf.html }\end{array}$ \\
\hline 394 & $15 / 3 / 2020$ & $\begin{array}{l}\text { http://hemeroteca.lavanguardia.com/preview/2020/03/15/pagina- } \\
\text { 8/295116593/pdf.html }\end{array}$ \\
\hline 395 & $15 / 3 / 2020$ & $\begin{array}{l}\text { http://hemeroteca.lavanguardia.com/preview/2020/03/15/pagina- } \\
\text { 10/295117019/pdf.html }\end{array}$ \\
\hline 396 & $15 / 3 / 2020$ & $\begin{array}{l}\text { http://hemeroteca.lavanguardia.com/preview/2020/03/15/pagina- } \\
\text { 11/295116912/pdf.html }\end{array}$ \\
\hline 397 & $15 / 3 / 2020$ & $\begin{array}{l}\text { http://hemeroteca.lavanguardia.com/preview/2020/03/15/pagina- } \\
\text { 11/295116912/pdf.html }\end{array}$ \\
\hline 398 & $15 / 3 / 2020$ & $\begin{array}{l}\text { http://hemeroteca.lavanguardia.com/preview/2020/03/15/pagina- } \\
\text { 14/295116946/pdf.html }\end{array}$ \\
\hline 399 & $15 / 3 / 2020$ & $\begin{array}{l}\text { http://hemeroteca.lavanguardia.com/preview/2020/03/15/pagina- } \\
\text { 15/295116941/pdf.html }\end{array}$ \\
\hline 400 & $15 / 3 / 2020$ & $\begin{array}{l}\text { http://hemeroteca.lavanguardia.com/preview/2020/03/15/pagina- } \\
\text { 16/295116786/pdf.html }\end{array}$ \\
\hline 401 & $15 / 3 / 2020$ & $\begin{array}{l}\text { http://hemeroteca.lavanguardia.com/preview/2020/03/15/pagina- } \\
\text { 18/295116670/pdf.html }\end{array}$ \\
\hline 402 & $15 / 3 / 2020$ & $\begin{array}{l}\text { http://hemeroteca.lavanguardia.com/preview/2020/03/15/pagina- } \\
\text { 19/295116648/pdf.html }\end{array}$ \\
\hline 403 & $15 / 3 / 2020$ & $\begin{array}{l}\text { http://hemeroteca.lavanguardia.com/preview/2020/03/15/pagina- } \\
\text { 22/295116543/pdf.html }\end{array}$ \\
\hline 404 & $15 / 3 / 2020$ & $\begin{array}{l}\text { http://hemeroteca.lavanguardia.com/preview/2020/03/15/pagina- } \\
\text { 24/295116267/pdf.html }\end{array}$ \\
\hline 405 & $15 / 3 / 2020$ & $\begin{array}{l}\text { http://hemeroteca.lavanguardia.com/preview/2020/03/15/pagina- } \\
\text { 24/295116267/pdf.html }\end{array}$ \\
\hline 406 & $15 / 3 / 2020$ & $\begin{array}{l}\text { http://hemeroteca.lavanguardia.com/preview/2020/03/15/pagina- } \\
\text { 25/295116244/pdf.html }\end{array}$ \\
\hline 407 & $15 / 3 / 2020$ & $\begin{array}{l}\text { http://hemeroteca.lavanguardia.com/preview/2020/03/15/pagina- } \\
\text { 28/295116993/pdf.html }\end{array}$ \\
\hline 408 & $15 / 3 / 2020$ & $\begin{array}{l}\text { http://hemeroteca.lavanguardia.com/preview/2020/03/15/pagina- } \\
\text { 29/295116983/pdf.html }\end{array}$ \\
\hline
\end{tabular}




\begin{tabular}{|c|c|c|}
\hline $\begin{array}{c}\text { Item ref. } \\
(*)\end{array}$ & Fecha & Enlace \\
\hline 409 & $15 / 3 / 2020$ & $\begin{array}{l}\text { http://hemeroteca.lavanguardia.com/preview/2020/03/15/pagina- } \\
\text { 29/295116983/pdf.html }\end{array}$ \\
\hline 410 & $15 / 3 / 2020$ & $\begin{array}{l}\text { http://hemeroteca.lavanguardia.com/preview/2020/03/15/pagina- } \\
\text { 30/295116978/pdf.html }\end{array}$ \\
\hline 411 & $15 / 3 / 2020$ & $\begin{array}{l}\text { http://hemeroteca.lavanguardia.com/preview/2020/03/15/pagina- } \\
\text { 30/295116978/pdf.html }\end{array}$ \\
\hline 412 & $15 / 3 / 2020$ & $\begin{array}{l}\text { http://hemeroteca.lavanguardia.com/preview/2020/03/15/pagina- } \\
\text { 31/295116998/pdf.html }\end{array}$ \\
\hline 413 & $15 / 3 / 2020$ & $\begin{array}{l}\text { http://hemeroteca.lavanguardia.com/preview/2020/03/15/pagina- } \\
\text { 32/295116485/pdf.html }\end{array}$ \\
\hline 414 & $15 / 3 / 2020$ & $\begin{array}{l}\text { http://hemeroteca.lavanguardia.com/preview/2020/03/15/pagina- } \\
\text { 33/295116729/pdf.html }\end{array}$ \\
\hline 415 & $15 / 3 / 2020$ & $\begin{array}{l}\text { http://hemeroteca.lavanguardia.com/preview/2020/03/15/pagina- } \\
\text { 34/295116496/pdf.html }\end{array}$ \\
\hline 416 & $15 / 3 / 2020$ & $\begin{array}{l}\text { http://hemeroteca.lavanguardia.com/preview/2020/03/15/pagina- } \\
\text { 36/295116397/pdf.html }\end{array}$ \\
\hline 417 & $15 / 3 / 2020$ & $\begin{array}{l}\text { http://hemeroteca.lavanguardia.com/preview/2020/03/15/pagina- } \\
\text { 58/295116411/pdf.html }\end{array}$ \\
\hline 418 & $15 / 3 / 2020$ & $\begin{array}{l}\text { http://hemeroteca.lavanguardia.com/preview/2020/03/15/pagina- } \\
\text { 60/295116620/pdf.html }\end{array}$ \\
\hline 419 & $15 / 3 / 2020$ & $\begin{array}{l}\text { http://hemeroteca.lavanguardia.com/preview/2020/03/15/pagina- } \\
\text { 62/295116616/pdf.html }\end{array}$ \\
\hline 420 & $15 / 3 / 2020$ & $\begin{array}{l}\text { http://hemeroteca.lavanguardia.com/preview/2020/03/15/pagina- } \\
\text { 62/295116616/pdf.html }\end{array}$ \\
\hline 421 & $15 / 3 / 2020$ & $\begin{array}{l}\text { http://hemeroteca.lavanguardia.com/preview/2020/03/15/pagina- } \\
\text { 63/295116628/pdf.html }\end{array}$ \\
\hline 422 & $15 / 3 / 2020$ & $\begin{array}{l}\text { http://hemeroteca.lavanguardia.com/preview/2020/03/15/pagina- } \\
\text { 63/295116628/pdf.html }\end{array}$ \\
\hline 423 & $15 / 3 / 2020$ & $\begin{array}{l}\text { http://hemeroteca.lavanguardia.com/preview/2020/03/15/pagina- } \\
\text { 64/295116455/pdf.html }\end{array}$ \\
\hline 424 & $15 / 3 / 2020$ & $\begin{array}{l}\text { http://hemeroteca.lavanguardia.com/preview/2020/03/15/pagina- } \\
\text { 67/295117011/pdf.html }\end{array}$ \\
\hline
\end{tabular}

(*): ver Anexo 2 
Fuente: "Diari ARA". Acceso disponible para subscriptores.

\begin{tabular}{|c|c|l|}
\hline $\begin{array}{c}\text { Items ref. } \\
\text { (*) }\end{array}$ & Fecha & \\
\hline $501-505$ & $15 / 2 / 2020$ & $\underline{\text { https://www.ara.cat/hemerotecadiari/20200215/ara/ }}$ \\
\hline $506-517$ & $16 / 2 / 2020$ & $\underline{\text { https://www.ara.cat/hemerotecadiari/20200216/ara/ }}$ \\
\hline $518-519$ & $17 / 2 / 2020$ & $\underline{\text { https://www.ara.cat/hemerotecadiari/20200217/ara/ }}$ \\
\hline $520-522$ & $18 / 2 / 2020$ & $\underline{\text { https://www.ara.cat/hemerotecadiari/20200218/ara/ }}$ \\
\hline $523-526$ & $19 / 2 / 2020$ & $\underline{\text { https://www.ara.cat/hemerotecadiari/20200219/ara/ }}$ \\
\hline 527 & $20 / 2 / 2020$ & $\underline{\text { https://www.ara.cat/hemerotecadiari/20200220/ara/ }}$ \\
\hline $528-530$ & $21 / 2 / 2020$ & $\underline{\text { https://www.ara.cat/hemerotecadiari/20200221/ara/ }}$ \\
\hline $531-538$ & $22 / 2 / 2020$ & $\underline{\text { https://www.ara.cat/hemerotecadiari/20200222/ara/ }}$ \\
\hline $539-542$ & $23 / 2 / 2020$ & $\underline{\text { https://www.ara.cat/hemerotecadiari/20200223/ara/ }}$ \\
\hline $543-547$ & $24 / 2 / 2020$ & $\underline{\text { https://www.ara.cat/hemerotecadiari/20200224/ara/ }}$ \\
\hline $548-555$ & $25 / 2 / 2020$ & $\underline{\text { https://www.ara.cat/hemerotecadiari/20200225/ara/ }}$ \\
\hline $556-566$ & $26 / 2 / 2020$ & $\underline{\text { https://www.ara.cat/hemerotecadiari/20200226/ara/ }}$ \\
\hline $567-577$ & $27 / 2 / 2020$ & $\underline{\text { https://www.ara.cat/hemerotecadiari/20200227/ara/ }}$ \\
\hline $578-588$ & $28 / 2 / 2020$ & $\underline{\text { https://www.ara.cat/hemerotecadiari/20200228/ara/ }}$ \\
\hline $589-599$ & $29 / 2 / 2020$ & $\underline{\text { https://www.ara.cat/hemerotecadiari/20200229/ara/ }}$ \\
\hline $600-613$ & $1 / 3 / 2020$ & $\underline{\text { https://www.ara.cat/hemerotecadiari/20200301/ara/ }}$ \\
\hline $614-618$ & $2 / 3 / 2020$ & $\underline{\text { https://www.ara.cat/hemerotecadiari/20200302/ara/ }}$ \\
\hline $619-627$ & $3 / 3 / 2020$ & $\underline{\text { https://www.ara.cat/hemerotecadiari/20200303/ara/ }}$ \\
\hline $628-636$ & $4 / 3 / 2020$ & $\underline{\text { https://www.ara.cat/hemerotecadiari/20200304/ara/ }}$ \\
\hline $637-647$ & $5 / 3 / 2020$ & $\underline{\text { https://www.ara.cat/hemerotecadiari/20200305/ara/ }}$ \\
\hline $648-655$ & $6 / 3 / 2020$ & $\underline{\text { https://www.ara.cat/hemerotecadiari/20200306/ara/ }}$ \\
\hline $656-666$ & $7 / 3 / 2020$ & $\underline{\text { https://www.ara.cat/hemerotecadiari/20200307/ara/ }}$ \\
\hline $667-671$ & $8 / 3 / 2020$ & $\underline{\text { https://www.ara.cat/hemerotecadiari/20200308/ara/ }}$ \\
\hline $672-679$ & $9 / 3 / 2020$ & $\underline{\text { https://www.ara.cat/hemerotecadiari/20200309/ara/ }}$ \\
\hline $680-697$ & $10 / 3 / 2020$ & $\underline{\text { https://www.ara.cat/hemerotecadiari/20200310/ara/ }}$ \\
\hline $698-721$ & $11 / 3 / 2020$ & $\underline{\text { https://www.ara.cat/hemerotecadiari/20200311/ara/ }}$ \\
\hline $722-746$ & $12 / 3 / 2020$ & $\underline{\text { https://www.ara.cat/hemerotecadiari/20200312/ara/ }}$ \\
\hline $747-780$ & $13 / 3 / 2020$ & $\underline{\text { https://www.ara.cat/hemerotecadiari/20200313/ara/ }}$ \\
\hline
\end{tabular}




\begin{tabular}{|c|c|l|}
\hline $\begin{array}{c}\text { Items ref. } \\
(*)\end{array}$ & Fecha & Enlace \\
\hline $781-816$ & $14 / 3 / 2020$ & $\underline{\text { https://www.ara.cat/hemerotecadiari/20200314/ara/ }}$ \\
\hline $817-846$ & $15 / 3 / 2020$ & $\underline{\text { https://www.ara.cat/hemerotecadiari/20200315/ara/ }}$ \\
\hline
\end{tabular}

(*): ver Anexo 2 